\title{
GALACTIC GLOBULAR AND OPEN CLUSTERS IN THE SLOAN DIGITAL SKY SURVEY. II. TEST OF THEORETICAL STELLAR ISOCHRONES
}

\author{
Deokkeun An ${ }^{1,10}$, Marc H. Pinsonneault ${ }^{1}$, Thomas Masseron ${ }^{1}$, Franck Delahaye ${ }^{2,3,4}$ Jennifer A. Johnson $^{1}$, \\ Donald M. TerndruP ${ }^{1,5}$, Timothy C. Beers ${ }^{6}$, Inese I. Ivans ${ }^{7,8}$, And ŽEluKo Ivezić 9 \\ ${ }^{1}$ Department of Astronomy, Ohio State University, 140 West 18th Avenue, Columbus, OH 43210, USA \\ ${ }^{2}$ Service d'Astrophysique, CEA/DSM/IRFU/SAp, CEA Saclay, 91191 Gif-sur-Yvette Cedex, France \\ ${ }^{3}$ Centre Lasers Intenses et Applications (CELIA), 351 Cours de la Libération, 33405 Talence Cedex, France \\ ${ }^{4}$ LERMA, Observatoire de Paris, CNRS, Université Paris Diderot, 5 place Jules Janssen, 92190 Meudon, France \\ ${ }^{5}$ Division of Astronomical Sciences, National Science Foundation, 4201 Wilson Boulevard, Arlington, VA 22230, USA \\ ${ }^{6}$ Department of Physics \& Astrophysics, CSCE: Center for the Study of Cosmic Evolution, and JINA: Joint Institute for Nuclear Astrophysics, Michigan State \\ University, E. Lansing, MI 48824, USA \\ ${ }^{7}$ The Observatories of the Carnegie Institution of Washington, 813 Santa Barbara Street, Pasadena, CA 91101, USA \\ ${ }^{8}$ Princeton University Observatory, Peyton Hall, Princeton, NJ 08544, USA \\ ${ }^{9}$ Department of Astronomy, University of Washington, Box 351580, Seattle, WA 98195, USA \\ Received 2008 December 15; accepted 2009 May 25; published 2009 July 2
}

\begin{abstract}
We perform an extensive test of theoretical stellar models for main-sequence (MS) stars in ugriz, using cluster fiducial sequences obtained in the previous paper of this series. We generate a set of isochrones using the Yale Rotating Evolutionary Code with updated input physics, and derive magnitudes and colors in ugriz from MARCS model atmospheres. These models match cluster MSs over a wide range of metallicity within the errors of the adopted cluster parameters. However, we find a large discrepancy of model colors at the lower MS ( $\left.T_{\text {eff }} \lesssim 4500 \mathrm{~K}\right)$ for clusters at and above solar metallicity. We also reach similar conclusions using the theoretical isochrones of Girardi et al. and Dotter et al., but our new models are generally in better agreement with the data. Using our theoretical isochrones, we also derive MS-fitting distances and turnoff ages for five key globular clusters, and demonstrate the ability to derive these quantities from photometric data in the Sloan Digital Sky Survey. In particular, we exploit multiple color indices $(g-r, g-i$, and $g-z)$ in the parameter estimation, which allows us to evaluate internal systematic errors. Our distance estimates, with an error of $\sigma_{(m-M)}=0.03-0.11 \mathrm{mag}$ for individual clusters, are consistent with Hipparcos-based subdwarf-fitting distances derived in the Johnson-Cousins or Strömgren photometric systems.
\end{abstract}

Key words: globular clusters: individual (M3, M5, M13, M15, M71, M92) - Hertzsprung-Russell (HR) diagram - open clusters and associations: individual (M67, NGC 6791) - stars: evolution - surveys

Online-only material: machine-readable table

\section{INTRODUCTION}

Improved stellar distance estimates are required to address many outstanding problems concerning the formation and evolution of the Galaxy. However, reliable distances from trigonometric parallaxes, such as those from the Hipparcos mission (ESA 1997), are restricted to a few hundred parsecs from the Sun. Therefore, for now, we must rely on indirect distance measurement techniques, such as photometric parallax or main-sequence (MS) fitting (e.g., Johnson 1957; Eggen \& Sandage 1959), to probe the spatial and kinematical (sub-)structures in the Galaxy (e.g., Jurić et al. 2008; Ivezić et al. 2008b).

Purely empirical photometric parallax relations can be constructed based on trigonometric parallaxes to nearby stars. However, the difficulty arises from a severely restricted sample of stars with good trigonometric parallaxes over a wide range of stellar mass and metallicity. In particular, colors and magnitudes of MS stars are sensitive to chemical content. Unfortunately, within the limited volume sampled by the Hipparcos mission, reliable trigonometric parallaxes are available for only a handful of metal-poor stars $([\mathrm{Fe} / \mathrm{H}]<-1.0)$, posing a challenge to Galactic halo studies relying on this information.

\footnotetext{
${ }^{10}$ Current address: Infrared Processing and Analysis Center, California Institute of Technology, Mail Stop 100-22, Pasadena, CA 91125, USA; deokkeun@ipac.caltech.edu.
}

A widely used technique for obtaining distances to individual stars and star clusters is to rely on theoretical predictions of stellar colors and magnitudes, tested with available observational constraints. Stellar evolutionary models predict luminosities and effective temperatures $\left(T_{\text {eff }}\right)$ as a function of time and chemical content (usually parameterized with $[\mathrm{Fe} / \mathrm{H}],[\alpha / \mathrm{Fe}]$, and helium abundance $Y$ ). Stellar atmosphere models are then used to transform theoretical quantities (luminosity and $T_{\text {eff }}$ ) to observables such as magnitudes and colors, expressed in terms of bolometric corrections and color $-T_{\text {eff }}$ relations. Stellar evolutionary models can be tested against the Sun, nearby stars with accurate parallaxes, and other stars, such as eclipsing binaries that have accurate masses and radii. Furthermore, multicolor photometry in nearby clusters and field stars can be used to test bolometric corrections and color $-T_{\text {eff }}$ relations (e.g., VandenBerg \& Clem 2003; Pinsonneault et al. 2004; An et al. 2007a, 2007b).

Pinsonneault et al. $(2003,2004)$ assessed the accuracy of theoretical stellar isochrones and reduced systematic errors in the model computation, particularly those arising from the transformation of theoretical to observational quantities. They demonstrated that stellar models from the Yale Rotating Evolutionary Code (YREC; Sills et al. 2000) are in good agreement with observed mass-luminosity- $T_{\text {eff }}$ relations for individual members $\left(4500 \lesssim T_{\text {eff }}(\mathrm{K}) \lesssim 6000\right)$ in the Hyades open cluster with accurate Hipparcos parallaxes (de Bruijne et al. 2001). However, they found that none of the widely used color $-T_{\text {eff }}$ relations in 
the Johnson-Cousins-Two Micron All Sky Survey (2MASS) broadband system (e.g., Alonso et al. 1995, 1996; Lejeune et al. 1997, 1998) could reproduce the observed shapes of the MS on color-magnitude diagrams (CMDs) for the Hyades open cluster. Since this implies problems with the adopted color $-T_{\text {eff }}$ relations, they introduced empirical corrections to the color- $T_{\text {eff }}$ relations in the models. An et al. (2007b) showed that these corrections improve the shape match to MSs of other open clusters, as well as the internal consistency of distances from several color indices.

In this paper we perform a test of theoretical isochrones in the Sloan Digital Sky Survey (SDSS; York et al. 2000; Stoughton et al. 2002; Abazajian et al. 2003, 2004, 2005; AdelmanMcCarthy et al. 2006, 2007, 2008) ugriz photometric system. Among previous and ongoing optical surveys, SDSS is the largest and most homogeneous database of stellar brightnesses currently available. SDSS measures the brightnesses of stars using a dedicated $2.5 \mathrm{~m}$ telescope (Gunn et al. 2006) in five broadband filters $u, g, r, i$, and $z$, with average wavelengths of $3551 \AA, 4686 \AA, 6165 \AA, 7481 \AA$, and $8931 \AA$, respectively (Fukugita et al. 1996; Stoughton et al. 2002). The imaging is carried out on moonless nights of good seeing (better than 1".6) under photometric conditions (Hogg et al. 2001). A portion of the sky (along great circles) is imaged in each run by six columns of CCDs (Gunn et al. 1998). Astrometric positions in SDSS are accurate to $<0^{\prime \prime} .1$ for sources with $r<20.5 \mathrm{mag}$ (Pier et al. 2003). However, the SDSS filters were not originally designed for stellar observations, owing to the primarily extragalactic mission of SDSS I. Therefore, it is important to understand the properties of stars in this system to exploit the full capabilities of the SDSS data set, especially for Galactic structure studies that require accurate distance measurements.

Galactic globular and open clusters provide an ideal opportunity to achieve this goal, because the same distance can be assumed for cluster members with a wide range of stellar masses. However, the standard SDSS photometric pipeline (Photo; Lupton et al. 2002) failed to provide photometry for the most crowded regions of high-density clusters. Photo was originally designed to handle high Galactic latitude fields with relatively low densities of field stars, and its photometry alone does not provide sufficiently well-defined cluster sequences.

In the previous paper of this series (An et al. 2008, hereafter Paper I), we employed the DAOPHOT/ALLFRAME (Stetson 1987, 1994) suite of programs to derive photometry for 17 globular clusters and three open clusters observed with SDSS. Our DAOPHOT photometry provides well-defined cluster sequences from the lower MS to the red giant branch (RGB). The DAOPHOT photometry is on the native SDSS $2.5 \mathrm{~m}$ system, so cluster fiducial sequences can be directly applied to other stars observed in SDSS, without relying upon any transformations. This will be of particular value for the study of the space motions of field stars in SDSS, where accurate distances are required in order to make full use of the available proper motions.

In light of this observational improvement, we assess the accuracy of theoretical isochrones that have been specifically constructed in the SDSS 2.5 m photometric system. Clem (2006) compared isochrones to the fiducial sequences for three globular clusters (M13, M71, and M92) and two open cluster (M67 and NGC 6791), in the primed $\left(u^{\prime} g^{\prime} r^{\prime} i^{\prime} z^{\prime}\right)$ filter system (see also Rider et al. 2004; Fornal et al. 2007). However, the $u^{\prime} g^{\prime} r^{\prime} i^{\prime} z^{\prime}$ system defined by the Smith et al. (2002) sample of standard stars is different from the natural ugriz system of the SDSS $2.5 \mathrm{~m}$ survey telescope (see Abazajian et al. 2003). Therefore, one must rely on transformation equations (Tucker et al. 2006), which limit the precision of the derived ugriz magnitudes.

We use several globular and open clusters that have been the subject of extensive studies in the literature. Distances to most of these clusters have been estimated based on photometry in the Johnson-Cousins system (e.g., Reid 1997, 1998; Gratton et al. 1997; Carretta et al. 2000, hereafter C00). Metal abundances for these clusters have also been obtained from high-resolution spectroscopy. In particular, a recent spectroscopic abundance study of Kraft \& Ivans (2003, hereafter KI03) provides an opportunity to check for possible systematic errors in cluster distance and age estimates from the cluster abundance scale, by comparison with those from Carretta \& Gratton (1997, hereafter CG97).

As a base case, we test stellar evolutionary models employed in the previous work (Pinsonneault et al. 2003, 2004; An et al. 2007a, 2007b) with updated input physics. We further inspect theoretical isochrones from two independent studies (Girardi et al. 2004; Dotter et al. 2008) to check theoretical uncertainties in the stellar interiors and atmosphere models. Theoretical color $-T_{\text {eff }}$ and bolometric corrections are also compared with those from different atmosphere models (Hauschildt et al. 1999; Castelli \& Kurucz 2003) to check theoretical uncertainties in the model atmospheres only.

Our goal in this study is to determine whether these models predict observed colors and magnitudes over a wide range of metallicity. In Section 2, we summarize the photometric accuracy of the DAOPHOT/ALLFRAME cluster photometry in Paper I and the cluster properties (distance, reddening, and metallicity) adopted in this study. In Section 3, we present a new set of stellar isochrones in ugriz and derive extinction coefficients. We also compare our models with those from Girardi et al. (2004) and Dotter et al. (2008). In Section 4, we test the accuracy of these models using cluster fiducial sequences for MS stars, and present empirical corrections on color $-T_{\text {eff }}$ relations for solar and super-solar metallicity clusters. In Section 5, we derive MS-fitting distances and turnoff ages for our sample globular clusters, and demonstrate the ability to derive these quantities from the SDSS photometric database, using our set of stellar isochrones.

\section{THE CLUSTER DATA}

\subsection{The Cluster Sample}

For the calibration of photometric parallax relations in any photometric filter system, it is important to select benchmark stellar systems with well established stellar properties. In particular, cluster distance, reddening, and metallicity estimates should be obtained reliably, independently from the photometric filter system in question. Although a few nearby open clusters have accurate geometric distance measurements (e.g., the Hyades and the Pleiades) in addition to precise reddening and metal abundance estimates, most of the stars in these clusters are too bright in SDSS because of the saturation limit in the survey ( $r \approx 14 \mathrm{mag}$ ).

In this study, we selected six globular clusters (M3, M5, M13, M15, M71, and M92) and two open clusters (M67 and NGC 6791) to test and calibrate theoretical stellar models. These clusters were included in the sample of the DAOPHOT/ ALLFRAME photometric data reduction in Paper I. For our sample open clusters, there exist reliable distances and metallicity estimates from high-resolution spectroscopy, as discussed in more detail below. 
The MS of many globular clusters in our sample are resolved 2-3 mag below the cluster MS turnoff (MSTO). They have spectroscopic metallicity estimates from CG97 and KI03, which are necessary to infer the absolute magnitudes of stars more accurately. Furthermore, distances to these clusters have been derived in the literature based on Hipparcos parallaxes to nearby subdwarfs, either in the Johnson-Cousins or Strömgren photometric systems. We therefore view these clusters as ideal tests of the color transformations for SDSS filters. We first review the DAOPHOT photometry in Paper I, and discuss cluster properties adopted in the model comparison (Section 4).

\subsection{Cluster Photometry and Fiducial Sequences}

Cluster photometry was obtained in Paper I from the SDSS ugriz imaging data, employing the DAOPHOT/ALLFRAME suite of programs. ${ }^{11}$ Due to the limited contamination from background stars, fiducial sequences of our sample clusters could be accurately derived on CMDs with several color indices $(u-g, g-r, g-i$, and $g-z)$ and $r$ as a luminosity index: hereafter $(u-g, r),(g-r, r),(g-i, r)$, and $(g-z, r)$, respectively.

The zero-point error of the DAOPHOT photometry is $\sim 1 \%$ $2 \%$, estimated from repeated flux measurements of stars in overlapping SDSS strips. The DAOPHOT magnitudes were tied to the Photo magnitude system using a set of cluster flanking fields, which are far away from the dense cluster cores. Error distributions at the bright ends indicate photometric random errors of $\sim 1 \%$ in griz and $\sim 2 \%$ in the $u$ band: see also Ivezić et al. (2004).

In Paper I we found that the zero points of the DAOPHOT photometry for M71 are uncertain, because of inaccurate Photo magnitudes in the cluster's flanking fields. For this reason, we adopted a fiducial sequence in $u^{\prime} g^{\prime} r^{\prime} i^{\prime} z^{\prime}$ from Clem et al. (2008). We included this cluster in the following analysis because M71 has an intermediate metal abundance $([\mathrm{Fe} / \mathrm{H}] \approx$ $-0.8)$, bridging the gap between globular and open clusters. We converted the Clem et al. fiducial sequences in the $u^{\prime} g^{\prime} r^{\prime} i^{\prime} z^{\prime}$ system onto the SDSS $2.5 \mathrm{~m}$ ugriz system, using the transformation equations in Tucker et al. (2006).

\subsection{Cluster Properties: Globular Clusters}

Table 1 lists $[\mathrm{Fe} / \mathrm{H}]$ estimates of clusters in the literature. In the model comparison, we consider metallicity scales for globular clusters from CG97 and KI03 (see also Kraft \& Ivans 2004). In each study a consistent technique was employed to derive metallicities for giants in all of our sample clusters. However, the CG97 values are based on Fe I lines, while the $\mathrm{KI} 03[\mathrm{Fe} / \mathrm{H}]$ values are based on Fe II lines from high-resolution spectra, which were argued to be less affected by non-local thermodynamic equilibrium (non-LTE) effects. KI03 found that their $[\mathrm{Fe} / \mathrm{H}]$ values are about 0.2 dex lower than those of CG97 and that they agree well with those of Zinn \& West (1984) for intermediate metallicities.

Table 2 lists distances for the globular clusters in our sample. The second column shows distances from the catalog of Harris (1996) 2003 February revision, which were primarily based on the mean $V$ magnitude of the horizontal branch (HB). The next five columns list distances derived from the Hipparcosbased subdwarf fitting in the literature (Reid 1997, 1998; C00; Grundahl et al. 2002; KI03). These studies used a subset of the Hipparcos subdwarfs to fit the MS of a cluster on CMDs,

\footnotetext{
11 Available at http://www.sdss.org/dr6/products/value_added/anjohnson08_ clusterphotometry.htm
}

Table 1

Sample Globular Clusters and $[\mathrm{Fe} / \mathrm{H}]$ Estimates in the Literature

\begin{tabular}{llcccr}
\hline \hline Name & NGC & Harris & Zinn \& West & CG97 $^{\mathrm{a}}$ & KI03 \\
\hline M15 & 7078 & -2.26 & -2.15 & -2.15 & -2.42 \\
M92 & 6341 & -2.28 & -2.24 & -2.15 & -2.38 \\
M13 & 6205 & -1.54 & -1.65 & -1.41 & -1.60 \\
M3 & 5272 & -1.57 & -1.66 & -1.34 & -1.50 \\
M5 & 5904 & -1.27 & -1.40 & -1.10 & -1.26 \\
M71 & 6838 & -0.73 & -0.58 & -0.70 & -0.81 \\
\hline
\end{tabular}

Note. ${ }^{a}$ Values are those shown in Table 1 of $\mathrm{C} 00$.

in either the Johnson-Cousins or the Strömgren photometric system. The quoted precision from Reid $(1997,1998)$ is shown in the table. We computed true distance moduli of clusters in C00, taking their adopted ratio of total to selective extinction $R_{V} \equiv A_{V} / E(B-V)=3.1$ and $E(B-V)$. The errors are the sum (in quadrature) of their fitting and total systematic errors $(0.12 \mathrm{mag})$. The sixth column shows the distance to M71 from Grundahl et al. (2002), who employed Strömgren uvby photometry for both subdwarfs and the cluster in MS fitting. Grundahl et al. found an apparent distance modulus $(m-M)_{V}=13.71 \pm 0.04$ (ran) \pm 0.07 (sys) for their adopted reddening of $E(B-V)=0.275$, which is translated into $(m-M)_{0}=12.86 \pm 0.08$. KI03 used the Hipparcos subdwarfs to derive distances to five globular clusters from their application of the MS-fitting technique. The last two columns in Table 2 list distances from MS fitting in this study, which are discussed in Section 5.1.

All of the above studies were based on a similar set of Hipparcos subdwarfs. However, there exist about a $10 \%$ difference in their distance estimates. This is because several systematic errors were involved in the subdwarf-fitting technique, such as the metallicity scale for subdwarfs and clusters, foreground reddening, effects of binaries, and the photometry used. Since no study has provided homogeneous distances to all of our sample clusters, we assumed the following subdwarf-fitting distances in the model comparison (Section 4): C00 (M5, M13, and M92), KI03 (M3 and M15), and Grundahl et al. (2002, M71).

We adopted the C00 distance estimates for M5, M13, and M92, since they employed the same metallicity scale for both subdwarfs and cluster stars in the subdwarf-fitting technique. Nevertheless, distances to M5 and M13 in C00 agree with Reid (1997), Reid (1998), and KI03, within their specified errors, although these studies adopted slightly different metallicity scales for both subdwarfs and clusters. Notable differences can be found for M92, where the Reid (1997) value is about 0.3 mag larger than the $\mathrm{C} 00$ estimate, and about 0.2 mag larger than the $\mathrm{KIO} 3$ value. As discussed in $\mathrm{C} 00$, a part of this discrepancy can be explained by the inhomogeneous metallicity scale used in Reid (1997) at the low-metallicity end. For this reason, we also adopted the distance to M15 from KI03. Although Grundahl et al. (2002) utilized the Strömgren $u v b y$ photometry to estimate the cluster metallicity, rather than high-resolution spectroscopy, they provided an internally more consistent metallicity scale for both subdwarfs and cluster stars than Reid (1998); see Figures 10 and 11 in Grundahl et al. (2002).

Table 3 summarizes reddening estimates for our sample globular clusters in the literature. The columns list values taken from the Harris compilation, the Schlegel et al. (1998) dust maps, and $E(B-V)$ estimates from KI03, who compared colors derived from high-resolution spectroscopic determinations of $T_{\text {eff }}$ with the observed colors of the same stars. There is 
Table 2

Distance Moduli of Globular Clusters

\begin{tabular}{|c|c|c|c|c|c|c|c|c|}
\hline \multirow[t]{2}{*}{ Cluster } & \multirow[t]{2}{*}{ Harris $^{\mathrm{a}}$} & \multicolumn{5}{|c|}{ Hipparcos-Based Subdwarf Fitting } & \multicolumn{2}{|c|}{ This Study } \\
\hline & & Reid (1997) & Reid (1998) & $\mathrm{COO} 0^{\mathrm{b}}$ & Grundahl et al. & $\mathrm{KI} 03$ & {$[\mathrm{Fe} / \mathrm{H}]_{\mathrm{CG} 97}$} & {$[\mathrm{Fe} / \mathrm{H}]_{\mathrm{KI} 03}$} \\
\hline M15 & 15.06 & $15.38 \pm 0.10$ & $\ldots$ & $\ldots$ & $\ldots$ & 15.25 & $15.14 \pm 0.11$ & $15.10 \pm 0.11$ \\
\hline M92 & 14.58 & $14.93 \pm 0.10$ & $\ldots$ & $14.64 \pm 0.14$ & $\ldots$ & 14.75 & $14.63 \pm 0.03$ & $14.66 \pm 0.04$ \\
\hline M13 & 14.42 & $14.48 \pm 0.10$ & $\ldots$ & $14.38 \pm 0.13$ & .. & 14.42 & $14.41 \pm 0.05$ & $14.34 \pm 0.05$ \\
\hline M3 & 15.09 & $\ldots$ & $\ldots$ & $\ldots$ & . . & 15.02 & $15.00 \pm 0.07$ & $14.95 \pm 0.07$ \\
\hline M5 & 14.37 & $14.45 \pm 0.10$ & $14.52 \pm 0.15$ & $14.46 \pm 0.13$ & $\ldots$ & 14.42 & $14.35 \pm 0.07$ & $14.26 \pm 0.06$ \\
\hline M71 & 13.02 & $\ldots$ & $13.19 \pm 0.15$ & $\ldots$ & $12.86 \pm 0.08$ & $\ldots$ & $12.96 \pm 0.08$ & $12.86 \pm 0.08$ \\
\hline
\end{tabular}

Notes.

${ }^{a}$ Distance moduli assuming $A_{V} / E(B-V)=3.1$ and reddening values from the catalog of Harris (1996) 2003 February revision.

${ }^{\mathrm{b}}$ Quadrature sum of systematic and random errors.

Table 3

Previous $E(B-V)$ Estimates for Globular Clusters

\begin{tabular}{lccc}
\hline \hline Cluster & Harris & Schlegel et al. $^{\mathrm{a}}$ & KI03 \\
\hline M15 & 0.10 & 0.11 & 0.10 \\
M92 & 0.02 & 0.02 & 0.02 \\
M13 & 0.02 & 0.02 & 0.02 \\
M3 & 0.01 & 0.01 & 0.01 \\
M5 & 0.03 & 0.04 & 0.03 \\
M71 & 0.25 & 0.32 & 0.32 \\
\hline
\end{tabular}

Note. ${ }^{\mathrm{a}}$ Quoted precision (16\%).

no appreciable offset in the mean between the three sets of estimates.

Since distances from MS fitting are sensitive to reddening, the same reddening values as in the individual subdwarf-fitting studies should be used in the model comparison. However, we adopted $E(B-V)$ estimates from KI03 for all of our sample globular clusters, because they provided homogeneous stellar $E(B-V)$ estimates from a consistent technique. Nevertheless, the $E(B-V)$ values adopted in $C 00$ are only 0.005 mag larger than those in KI03 for M5 and M92, with the same $E(B-V)$ for M13. In addition, with the exception of M71, the difference between the KI03 values and those of the dust map values from Schlegel et al. (1998) is $0.004 \pm 0.005 \mathrm{mag}$ in the sense of Schlegel et al. (1998) minus KI03. In the case of M71, we adopted the same $E(B-V)=0.28$ as in Grundahl et al. (2002). We assumed a $20 \%$ error in $E(B-V)$ in the model comparison (Section 4) for all clusters in our sample, which encompasses the differences between the studies shown in Table 3 .

\subsection{Cluster Properties: Open Clusters}

Table 4 lists metallicity, reddening, and distance estimates for our sample open clusters. For M67 we took the average redden- ing and metallicity estimates from high-resolution spectroscopy in the literature (An et al. 2007b, and references therein). Although M67 is closer than any other cluster in this study, it is still too far away for Hipparcos to provide a direct distance measurement. We adopted a cluster distance estimated from an empirically calibrated set of isochrones in the JohnsonCousins-2MASS system (An et al. 2007b). In that paper, we noted that the application of these isochrones resulted in consistent estimates for metallicity and reddening with those in the literature.

For NGC 6791 there exist four recent $[\mathrm{Fe} / \mathrm{H}]$ measurements from high-resolution spectroscopic studies: $[\mathrm{Fe} / \mathrm{H}]=$ $+0.47 \pm 0.04$ (random) \pm 0.08 (systematic; Gratton et al. 2006), $+0.39 \pm 0.01$ (random) (Carraro et al. 2006, $+0.35 \pm 0.02$ (random) \pm 0.1 (systematic; Origlia et al. 2006), and +0.40 (Jensen et al. 2006). In the model comparison (Section 4), we adopted the average of these measurements $\langle[\mathrm{Fe} / \mathrm{H}]\rangle=+0.40 \pm 0.03$ with the same weight given to each study. Using an extended set of calibrated isochrones in the Johnson-Cousins-2MASS system, we derived a photometric metallicity, $[\mathrm{Fe} / \mathrm{H}]=+0.43 \pm 0.07$ (M. H. Pinsonneault \& D. An 2009, in preparation), that is consistent with the above measurements, assuming solar abundance mixtures (see Gratton et al. 2006; Origlia et al. 2006 for subsolar [C/Fe]). However, we found $E(B-V)=0.098 \pm 0.014$ from the calibrated isochrones, while reddening estimates in the literature vary between $0.1 \lesssim E(B-V) \lesssim 0.2$. We adopted our $E(B-V)$ in the model comparison. Both our photometric metallicity and reddening values yield a cluster distance modulus, $(m-M)_{0}=13.02 \pm 0.05$, assuming solar abundance mixtures. If we use our adopted metallicity $[\mathrm{Fe} / \mathrm{H}]=+0.40$ in this study, the distance modulus decreases by $\approx 0.03 \mathrm{mag}$. Given the size of the $1 \sigma$ error in distance, however, we neglected this difference, and adopted $(m-M)_{0}=13.02 \pm 0.05$ for the model comparison.

Table 4

Properties of Open Clusters

\begin{tabular}{|c|c|c|c|c|c|c|c|}
\hline \multirow[t]{2}{*}{ NGC } & \multirow{2}{*}{$\begin{array}{c}\text { Alternate } \\
\text { Name }\end{array}$} & \multirow{2}{*}{$\begin{array}{l}\text { High Resolution } \\
{[\mathrm{Fe} / \mathrm{H}]}\end{array}$} & \multicolumn{2}{|c|}{$E(B-V)$} & \multicolumn{2}{|c|}{$(m-M)_{0}$} & \multirow[t]{2}{*}{ References } \\
\hline & & & Previous Work & Schlegel et al. ${ }^{\mathrm{a}}$ & Previous Work & This Study & \\
\hline 2682 & M67 & $+0.00 \pm 0.01$ & $0.041 \pm 0.004$ & 0.032 & $9.61 \pm 0.03$ & $9.59 \pm 0.03$ & 1 \\
\hline 6791 & & $+0.40 \pm 0.03$ & $0.098 \pm 0.014$ & 0.155 & $13.02 \pm 0.05$ & $12.95 \pm 0.05$ & 2 \\
\hline
\end{tabular}

References. for high-resolution spectroscopic $[\mathrm{Fe} / \mathrm{H}]$ values in the literature, and estimates for stellar $E(B-V)$ and $(m-M)_{0}:(1)$ An et al. (2007b), and references therein; (2) M. H. Pinsonneault \& D. An (2009, in preparation), and references therein.

${ }^{a}$ Quoted precision (16\%). 


\section{MODELS}

In this section we generate a set of theoretical isochrones in ugriz, which serves as a base case for the comparison with cluster fiducial sequences (Section 4) and in the estimation of distances and ages of globular clusters (Section 5). We further use other theoretical isochrones and color $-T_{\text {eff }}$ relations in the literature to inspect theoretical uncertainties in the model computations.

\subsection{Theoretical Isochrones in ugriz}

Stellar evolutionary tracks were generated using YREC (Sills et al. 2000; Delahaye \& Pinsonneault 2006) for a wide range of compositions and ages. Similar models were employed in our previous studies in the Johnson-Cousins-2MASS filter system (Pinsonneault et al. 2003, 2004; An et al. 2007a, 2007b), but the new models include updated input physics (see below). Details of the interiors models will be presented in a paper in preparation.

We recently adopted atomic opacity data from the Opacity Project (OP; Badnell et al. 2005) for our models. Previous models used the OPAL opacities (Iglesias \& Rogers 1996). In our view the OP data have benefited from improvements in the equation of state, especially for iron, and the availability of the monochromatic opacities permits tables to be readily constructed for a wider range of mixtures than for OPAL. See Badnell et al. (2005) for a comparison of the underlying opacities and a discussion of the differences in the underlying physical model. For $T<10^{4} \mathrm{~K}$ we used the molecular opacities of Alexander \& Ferguson (1994). We recognize that there is an inconsistency between the mixture of heavy elements in these tables and the Grevesse \& Sauval (1998) mix adopted in this paper, but the mixtures are quite similar, and the impact on the opacities is typically at the $0.01-0.03$ dex level for solar metallicity. We used the OPAL 2001 equation of state (Rogers \& Nayfonov 2002) for $T>10^{6} \mathrm{~K}$ and the Saumon et al. (1995) equation of state for $T<10^{5.5} \mathrm{~K}$. In the transition region between these two temperatures, both formulations are weighted with a ramp function and averaged. The chemical composition of each shell was updated using nuclear reaction rates in Bahcall et al. (2001). The models do not include gravitational settling. A mixing length of $\alpha=1.78$ and the solar helium abundance $Y_{\odot}=0.269$ were calibrated by matching the solar radius $\left(R_{\odot}=\right.$ $\left.6.9598 \times 10^{8} \mathrm{~m}\right)$ and luminosity $\left(L_{\odot}=3.8418 \times 10^{33} \mathrm{erg} \mathrm{s}^{-1}\right)$ at the age of the Sun $(4.57 \mathrm{Gyr})$; see Delahaye \& Pinsonneault (2006). We used the ATLAS9 atmosphere models to set the surface boundary condition, defined as the pressure at $\tau=2 / 3$.

At $[\alpha / \mathrm{Fe}]=0$, we used the solar mixture of Grevesse \& Sauval (1998) for the initial chemical mixture, and generated models at $[\mathrm{Fe} / \mathrm{H}]=-3.0$, from $[\mathrm{Fe} / \mathrm{H}]=-2.0$ to $[\mathrm{Fe} / \mathrm{H}]=$ -0.5 with intervals of $\Delta[\mathrm{Fe} / \mathrm{H}]=0.25$, from $[\mathrm{Fe} / \mathrm{H}]=-0.5$ to $[\mathrm{Fe} / \mathrm{H}]=0.5$ with intervals of $\Delta[\mathrm{Fe} / \mathrm{H}]=0.1$, and $[\mathrm{Fe} / \mathrm{H}]=0.75$. We assumed the helium enrichment parameter, $\Delta Y / \Delta Z=1.2$, along with the primordial helium abundance, $Y_{p}=0.245 \pm 0.002$ (see Pinsonneault et al. 2003). ${ }^{12}$ We also computed $\alpha$-element enhanced models at $[\alpha / \mathrm{Fe}]=+0.3$ and +0.6 for each $[\mathrm{Fe} / \mathrm{H}]$, by increasing the ratio of $\alpha$ elements $(\mathrm{O}, \mathrm{Ne}, \mathrm{Mg}, \mathrm{Si}, \mathrm{S}, \mathrm{Ar}, \mathrm{Ca}$, and $\mathrm{Ti})$ by the same amount in the Grevesse \& Sauval (1998) mixture using OP tables computed with the same mixture. We followed the evolution of each model from pre-MS until hydrogen in the core is exhausted.

\footnotetext{
12 We assumed that the helium abundance is a function of the heavy-element
} content $Z$ as given by $Y=Y_{p}+(\Delta Y / \Delta Z) Z$.
Table 5

Bolometric Corrections and Synthetic Colors in ugriz from the MARCS Model Atmospheres

\begin{tabular}{lcccccccc}
\hline \hline $\begin{array}{l}{[\mathrm{Fe} / \mathrm{H}]} \\
(\mathrm{dex})\end{array}$ & $\begin{array}{c}{[\alpha / \mathrm{Fe}]} \\
(\mathrm{dex})\end{array}$ & $\begin{array}{c}T_{\mathrm{eff}} \\
(\mathrm{K})\end{array}$ & $\begin{array}{c}\log g \\
(\mathrm{dex})\end{array}$ & $\begin{array}{c}\mathrm{BC}(r) \\
(\mathrm{mag})\end{array}$ & $\begin{array}{c}u-g \\
(\mathrm{mag})\end{array}$ & $\begin{array}{c}g-r \\
(\mathrm{mag})\end{array}$ & $\begin{array}{c}g-i \\
(\mathrm{mag})\end{array}$ & $\begin{array}{c}g-z \\
(\mathrm{mag})\end{array}$ \\
\hline-3.0 & 0.4 & 4000 & 3.0 & -0.398 & 2.037 & 1.115 & 1.584 & 1.867 \\
-3.0 & 0.4 & 4000 & 3.5 & -0.374 & 1.988 & 1.111 & 1.579 & 1.859 \\
-3.0 & 0.4 & 4000 & 4.0 & -0.354 & 1.967 & 1.116 & 1.583 & 1.860 \\
-3.0 & 0.4 & 4000 & 4.5 & -0.333 & 1.973 & 1.127 & 1.592 & 1.866 \\
-3.0 & 0.4 & 4000 & 5.0 & -0.306 & 1.999 & 1.146 & 1.609 & 1.878 \\
-3.0 & 0.4 & 4000 & 5.5 & -0.275 & 2.057 & 1.169 & 1.629 & 1.894 \\
\hline
\end{tabular}

Notes. The AB corrections for the SDSS magnitudes and empirical color corrections are not applied.

(This table is available in its entirety in a machine-readable form in the online journal. A portion is shown here for guidance regarding its form and content.)

A discussion of the post-MS evolutionary tracks is outside the scope of this paper.

Theoretical isochrones in the luminosity- $T_{\text {eff }}$ plane were then transformed onto the observed CMD plane, using a set of synthetic spectra. We used MARCS (Gustafsson et al. 2008) to construct stellar atmosphere models over a wide range of $T_{\text {eff }}$ (from $4000 \mathrm{~K}$ to $8000 \mathrm{~K}$ with intervals of $250 \mathrm{~K}$ ), $\log g$ (from 3.5 dex to 5.5 dex with intervals of $0.5 \mathrm{dex}$ ), and chemical abundances (see below). These models are based on onedimensional plane-parallel geometry, hydrostatic equilibrium, mixing-length theory (MLT) convection, and LTE. Microturbulence $(\xi)$ was chosen to be $2.0 \mathrm{~km} \mathrm{~s}^{-1}$. We assumed the same $[\alpha / \mathrm{Fe}]$ abundance scales (Grevesse \& Sauval 1998) as those in the interior model computations. Based on these models, we constructed synthetic spectra at $[\mathrm{Fe} / \mathrm{H}]([\alpha / \mathrm{Fe}])=-3.0(0.4)$, $-2.0(0.0,0.4),-1.5(0.0,0.4),-1.0(0.0,0.4),-0.5(0.0,0.2)$, $-0.3(0.0,0.1),-0.2(0.0,0.1),-0.1(0.0), 0.0(0.0),+0.1(0.0)$, $+0.2(0.0),+0.4(0.0)$.

Stellar magnitudes were derived by convolving synthetic spectra with the SDSS ugriz filter response curves, ${ }^{13}$ which include extinction through an air mass of 1.3 at Apache Point Observatory. We integrated flux with weights given by photon counts (Girardi et al. 2002). Magnitudes were then put onto the $\mathrm{AB}$ magnitude system using a flat $3631 \mathrm{Jy}$ spectrum (Oke \& Gunn 1983). Bolometric corrections in the $r$ band and synthetic colors are presented in Table 5, where we adopted the bolometric magnitude of the Sun, $M_{\mathrm{bol}, \odot}=4.74 \mathrm{mag}$ (Bessell et al. 1998).

In the following analysis, we adopted an $\alpha$-element enhancement scheme motivated by the observational nature of these elements among field and cluster stars from high-resolution spectroscopic studies, as summarized by Venn et al. (2004) and Kirby et al. (2008): $[\alpha / \mathrm{Fe}]=+0.4$ at $[\mathrm{Fe} / \mathrm{H}]=-3.0,[\alpha / \mathrm{Fe}]$ $=+0.3$ at $[\mathrm{Fe} / \mathrm{H}]=-2.0,-1.5,-1.0,[\alpha / \mathrm{Fe}]=+0.2$ at $[\mathrm{Fe} /$ $\mathrm{H}]=-0.5$, and $[\alpha / \mathrm{Fe}]=+0.0$ at $[\mathrm{Fe} / \mathrm{H}]=-0.3,-0.2,-0.1$, $+0.0,+0.1,+0.2,+0.4$. We used a linear interpolation in this metallicity grid to obtain isochrones at an intermediate $[\mathrm{Fe} / \mathrm{H}]$ value. As an alternate case, we also tested models with $[\alpha / \mathrm{Fe}]$ $=+0.4$ at $[\mathrm{Fe} / \mathrm{H}]=-2.0,-1.5,-1.0$, given a $\Delta[\alpha / \mathrm{Fe}] \sim \pm 0.1$ dispersion in the observed distribution of $[\alpha / \mathrm{Fe}]$.

Isochrones constructed in this way are on a perfect $\mathrm{AB}$ magnitude system, in which magnitudes can be translated directly into physical flux units. However, it is known that the SDSS photometry slightly deviates from a true AB system

\footnotetext{
13 http://www.sdss.org/dr6/instruments/imager/
} 
(Abazajian et al. 2004). To compare our models with fiducial sequences, we adjusted model magnitudes using $\mathrm{AB}$ corrections given by Eisenstein et al. (2006): $u_{\mathrm{AB}}=u-0.040, i_{\mathrm{AB}}=$ $i+0.015$, and $z_{\mathrm{AB}}=z+0.030$, with no corrections in $g$ and $r$ (see also Holberg \& Bergeron 2006).

The SDSS magnitudes are on the asinh magnitude system (Lupton et al. 1999), which is essentially the same as the standard astronomical magnitude at high signal-to-noise ratios. ${ }^{14}$ Near the $95 \%$ detection repeatability limit for point sources in SDSS $(u=22.0 \mathrm{mag}, g=22.2 \mathrm{mag}, r=22.2 \mathrm{mag}$, $i=21.3 \mathrm{mag}$, and $z=20.5 \mathrm{mag}$ ), the difference between the asinh and Pogson magnitudes are $\leqslant 0.01 \mathrm{mag}$, and it rapidly becomes zero as a source becomes brighter. Since our fiducial sequences were derived using relatively bright stars (Paper I), we compare theoretical isochrones on the Pogson system directly with the DAOPHOT cluster photometry on the asinh system, without relying upon any transformation.

The isochrones constructed in this way are available at http://www.astronomy.ohio-state.edu/iso/sdss.html. In the following analysis, we refer to our models as YREC+MARCS to distinguish from the other theoretical isochrones described in Section 3.3.

\subsection{Extinction Coefficients}

We derived extinction coefficients in the ugriz filters using MARCS synthetic spectra. We first reddened the synthetic spectra assuming the Cardelli et al. (1989) extinction law, which is given in polynomial equations for the optical wavelength range as a function of the wavelength and $R_{V}$. We then convolved the reddened spectra with the SDSS filter responses, over a wide range of stellar parameters and $R_{V}$. In addition to the ugriz filter response functions, we also used the normalized $V$ filter response from Bessell (1990) to compute the absorption ratios of SDSS filters with respect to the Johnson $V$ band, $A_{\Lambda} / A_{V}$, where $\Lambda$ represents one of the SDSS filters.

For solar metallicity models with $T_{\text {eff }}=5750 \mathrm{~K}$ and $\log g=4.5$, we found $A_{u} / A_{V}=1.567 \pm 0.044$, $A_{g} / A_{V}=1.196 \pm 0.013, A_{r} / A_{V}=0.874 \pm 0.005, A_{i} / A_{V}=$ $0.672 \pm 0.011$, and $A_{z} / A_{V}=0.488 \pm 0.014$, assuming $R_{V}=$ 3.1. The errors represent the case of assuming $\Delta R_{V}= \pm 0.2$. Although there exists a mild variation as a function of colors or $T_{\text {eff }}$ of models, differences are within $\approx 1 \sigma$ of the above estimates. In this paper we neglected these color-dependent variations in extinction ratios, since most of the sample clusters have interstellar reddening less than $E(B-V) \sim 0.1$.

Girardi et al. (2004) also estimated extinction coefficients based on the same reddening law (Cardelli et al. 1989), but using synthetic ATLAS9 spectra. Our values are in good agreement with their values. At $4000 \leqslant T_{\text {eff }}(\mathrm{K}) \leqslant 8000$ with $\log g=4.5$, the differences are only $\Delta A_{\Lambda} / A_{V} \sim 0.01$.

We additionally derived extinction coefficients in $u^{\prime} g^{\prime} r^{\prime} i^{\prime} z^{\prime}$ for the fiducial sequence of M71 (Clem et al. 2008), since transformations from $u g r i z$ to $u^{\prime} g^{\prime} r^{\prime} i^{\prime} z^{\prime}$ were derived for unreddened colors (Tucker et al. 2006). We derived extinction coefficients for USNO $u^{\prime} g^{\prime} r^{\prime} i^{\prime} z^{\prime}$ filters ${ }^{15}$ using solar metallicity models with $T_{\text {eff }}=5750 \mathrm{~K}$ and $\log g=4.5$ at $R_{V}=3.1: A_{u^{\prime}} / A_{V}=1.569$, $A_{g^{\prime}} / A_{V}=1.177, A_{r^{\prime}} / A_{V}=0.866, A_{i^{\prime}} / A_{V}=0.656$, and $A_{z^{\prime}} / A_{V}=0.488$.

\footnotetext{
14 see also http://www.sdss.org/dr6/algorithms/fluxcal.html

15 See http://www-star.fnal.gov/ugriz/Filters/response.html
}

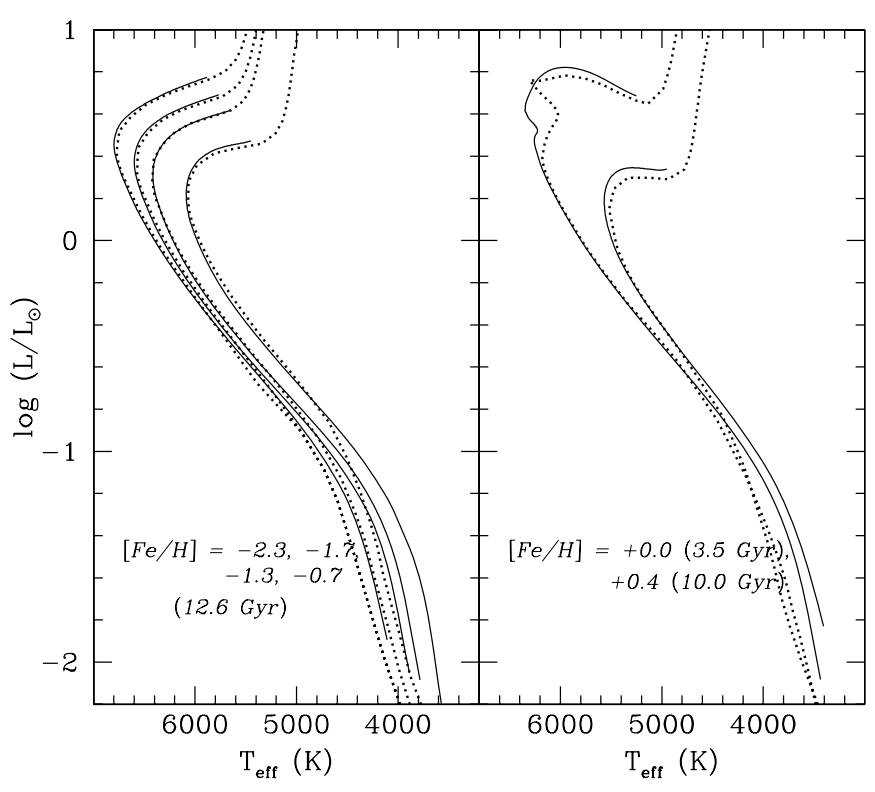

Figure 1. Comparison between Padova (dotted line) and YREC (solid line) evolutionary models. Left: isochrones are shown at $[\mathrm{Fe} / \mathrm{H}]=-2.32,-1.71$, -1.31 , and -0.71 , with $[\alpha / \mathrm{Fe}]=0.0$. Ages of $12.6 \mathrm{Gyr}$ are assumed. Right: isochrones are shown at $[\mathrm{Fe} / \mathrm{H}]=0.0(3.5 \mathrm{Gyr})$ and $+0.37(10.0 \mathrm{Gyr})$ with $[\alpha / \mathrm{Fe}]=0.0$.

For a given $R_{V}$ and $E(B-V)$ of a cluster, we computed extinction in the $r$ band and color-excess values in ugriz from

$$
A_{r}=\left(\frac{A_{r}}{A_{V}}\right) E(B-V) R_{V},
$$

and

$$
E\left(\Lambda_{1}-\Lambda_{2}\right)=\left(\frac{A_{\Lambda_{1}}}{A_{V}}-\frac{A_{\Lambda_{2}}}{A_{V}}\right) E(B-V) R_{V},
$$

where $\Lambda_{1}$ and $\Lambda_{2}$ represent each of the SDSS filter passbands.

\subsection{Other Models: Isochrones}

Isochrones in ugriz are also available from two other independent studies. Girardi et al. (2004) provided the first extensive set of isochrones in ugriz filters, based on evolutionary models used in their earlier studies (see Girardi et al. 2002). To derive bolometric corrections and color- $T_{\text {eff }}$ relations, they have mainly used ATLAS9 non-overshooting models (Castelli et al. 1997; Bessell et al. 1998); hereafter we refer to these isochrones as Padova+ATLAS9. On the other hand, Dotter et al. (2008) recently presented the Dartmouth Stellar Evolution Program (DSEP) isochrones, where PHOENIX model atmospheres (Hauschildt et al. 1999) were employed to convert luminosity and $T_{\text {eff }}$ into magnitudes and colors; hereafter we refer to these isochrones as DSEP+PHOENIX. In this section, we compare different evolutionary models $\left(L-T_{\text {eff }}\right)$ and atmosphere models (bolometric corrections and color- $T_{\text {eff }}$ relations) separately.

Figure 1 shows the comparison between Padova (dotted lines) and YREC (solid) isochrones on the $\log \left(L / L_{\odot}\right)-T_{\text {eff }}$ plane. Padova isochrones are shown at $Z=0.0001([\mathrm{Fe} / \mathrm{H}]=-2.32)$, $Z=0.0004([\mathrm{Fe} / \mathrm{H}]=-1.71), Z=0.001([\mathrm{Fe} / \mathrm{H}]=-1.31)$, $Z=0.004([\mathrm{Fe} / \mathrm{H}]=-0.71), Z=0.019([\mathrm{Fe} / \mathrm{H}]=+0.00)$, and $Z=0.040([\mathrm{Fe} / \mathrm{H}]=+0.37)$, taking their adopted solar composition $(Y=0.273$ and $Z=0.019)$ to compute $[\mathrm{Fe} / \mathrm{H}]$ values. All of these models are based on the solar abundance 


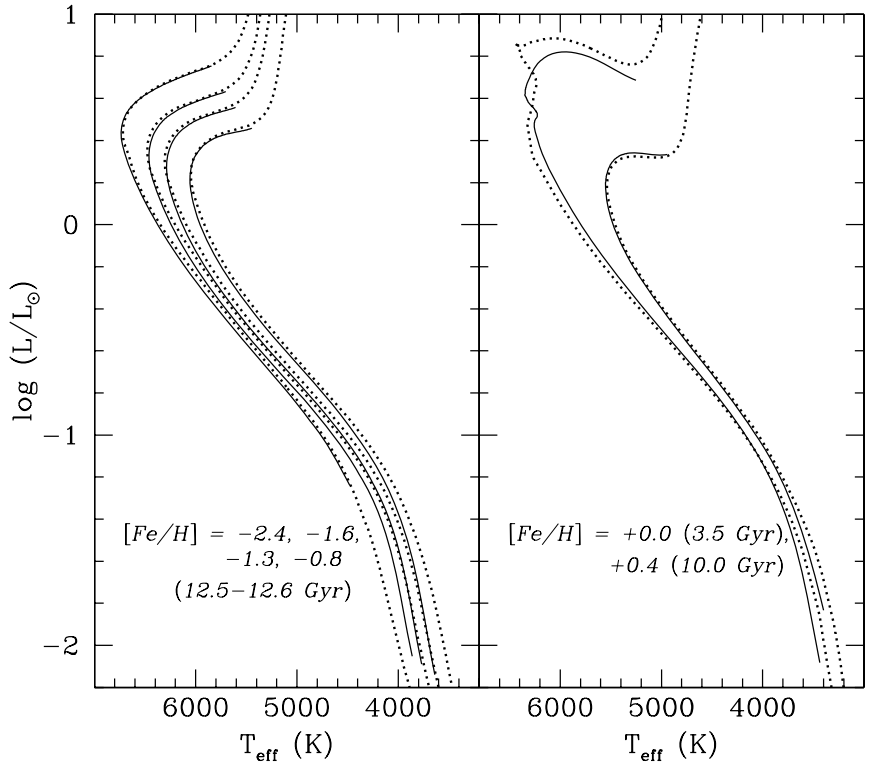

Figure 2. Comparison between DSEP (dotted line) and YREC (solid line) evolutionary models. Left: isochrones are shown at $[\mathrm{Fe} / \mathrm{H}]=-2.42,-1.60$, -1.26 , and -0.81 , with $\alpha$-element enhancement adopted in this study $([\alpha / \mathrm{Fe}] \approx+0.3$ at $[\mathrm{Fe} / \mathrm{H}] \lesssim-1$; see Section 3.1). Ages of $12.6 \mathrm{Gyr}$ are assumed. Right: isochrones are shown at $[\mathrm{Fe} / \mathrm{H}]=0.00(3.5 \mathrm{Gyr})$ and +0.40 $(10.0 \mathrm{Gyr})$ with $[\alpha / \mathrm{Fe}]=0.0$.

ratio, since Girardi et al. (2004) do not provide models with $\alpha$-element enhancement.

As shown in Figure 1, YREC and Padova isochrones are generally in good agreement with each other, but they exhibit a large difference at the lower MS, in the sense that the Padova models are hotter than the YREC models for a given luminosity. This difference is likely due to the use of different equations of state at low temperatures. Padova models use those in Mihalas et al. (1990), while YREC models adopt the Saumon et al. (1995) equation of state. The morphologies of the MSTOs differ for the solar metallicity, 3.5 Gyr models, because Padova models include convective core overshooting (see Girardi et al. 2000).

Figure 2 shows a similar comparison in the $\log \left(L / L_{\odot}\right)-T_{\text {eff }}$ plane between DSEP (dotted line) and YREC (solid line), both of which include $\alpha$-element enhancement. DSEP isochrones at intermediate $[\alpha / \mathrm{Fe}]$ values were obtained by interpolating models over their customized grid at $[\alpha / \mathrm{Fe}]=0.0,0.2$, and 0.4 . In the left-hand panel, these isochrones are shown at $[\mathrm{Fe} / \mathrm{H}]=$ $-2.42,-1.60,-1.26$, and -0.81 , with our adopted $\alpha$-element enhancement scheme $([\alpha / \mathrm{Fe}] \approx+0.3$ at $[\mathrm{Fe} / \mathrm{H}] \lesssim-1$; see Section 3.1). In the right-hand panel, isochrones are shown at $[\mathrm{Fe} / \mathrm{H}]=+0.0$ and +0.4 with no $\alpha$-element enhancement.

As shown in Figure 2, the two models are generally in good agreement with each other on the $\log \left(L / L_{\odot}\right)$ versus $T_{\text {eff }}$ plane. In fact, DSEP has been developed based on a similar code to YREC (Demarque et al. 2007), and many input ingredients are the same as in YREC. However, they have been subsequently modified by the two independent groups. For example, DSEP includes the PHOENIX model atmosphere to set the surface boundary condition, while YREC adopts the ATLAS9 model. DSEP uses high-temperature opacities from OPAL (Iglesias \& Rogers 1996), while YREC utilizes OP data (Badnell et al. 2005). As mentioned above, the different MSTO morphology of $4 \mathrm{Gyr}$, solar metallicity isochrones is due to the inclusion/ exclusion of the convective core overshoot in DSEP/YREC.

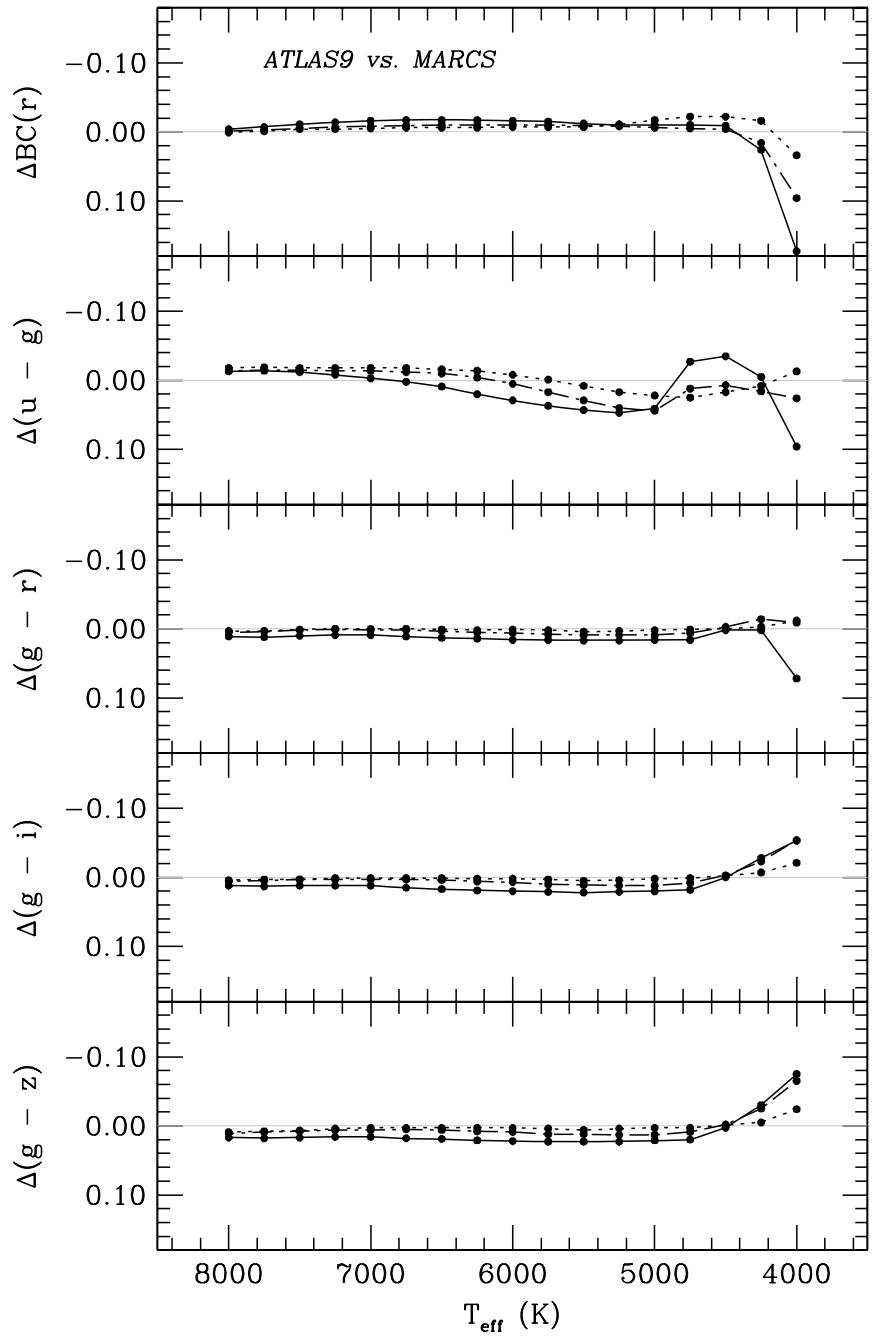

Figure 3. Comparisons in $r$-band bolometric corrections and synthetic colors between ATLAS9 and MARCS atmosphere models, in the sense of ATLAS9 minus MARCS. Comparisons are shown at $\log g=4.0$, with three different metallicities: $[\mathrm{Fe} / \mathrm{H}]=-2.0$ and $[\alpha / \mathrm{Fe}]=0.4$ (dotted line), $[\mathrm{Fe} / \mathrm{H}]=-1.0$ and $[\alpha / \mathrm{Fe}]=0.4$ (dot-dashed line), $[\mathrm{Fe} / \mathrm{H}]=+0.0$ and $[\alpha / \mathrm{Fe}]=0.0$ (solid line).

\subsection{Other Models: Bolometric Corrections and Color- $T_{\text {eff }}$ Relations}

Figure 3 compares $r$-band bolometric corrections and synthetic colors from ATLAS9 ${ }^{16}$ and MARCS, in the sense of the former minus the latter. We derived magnitudes and colors for ATLAS9 spectra, following the same steps as for MARCS. Comparisons are shown at $\log g=4.0$ with three different metallicities: $[\mathrm{Fe} / \mathrm{H}]=-2.0$ and $[\alpha / \mathrm{Fe}]=0.4$ (dotted line), $[\mathrm{Fe} / \mathrm{H}]=-1.0$ and $[\alpha / \mathrm{Fe}]=0.4$ (dot-dashed line), $[\mathrm{Fe} / \mathrm{H}]=+0.0$ and $[\alpha / \mathrm{Fe}]=0.0$ (solid line). Although the difference between ATLAS9 and MARCS is becoming larger at a lower $T_{\text {eff }}$ end in the comparison, the agreement between these two models is generally good $(\lesssim 0.02$ mag in bolometric corrections and colors) at $5000 \lesssim T_{\text {eff }}(\mathrm{K}) \lesssim 8000$. The comparison in $u-g$ suggests a large theoretical uncertainty in colors that involve the $u$ bandpass.

Similarly, Figure 4 shows comparisons in $r$-band bolometric corrections and synthetic colors between MARCS and the LTE-

\footnotetext{
$\overline{16} \mathrm{http}: / /$ wwwuser.oat.ts.astro.it/castelli/grids.html
} 


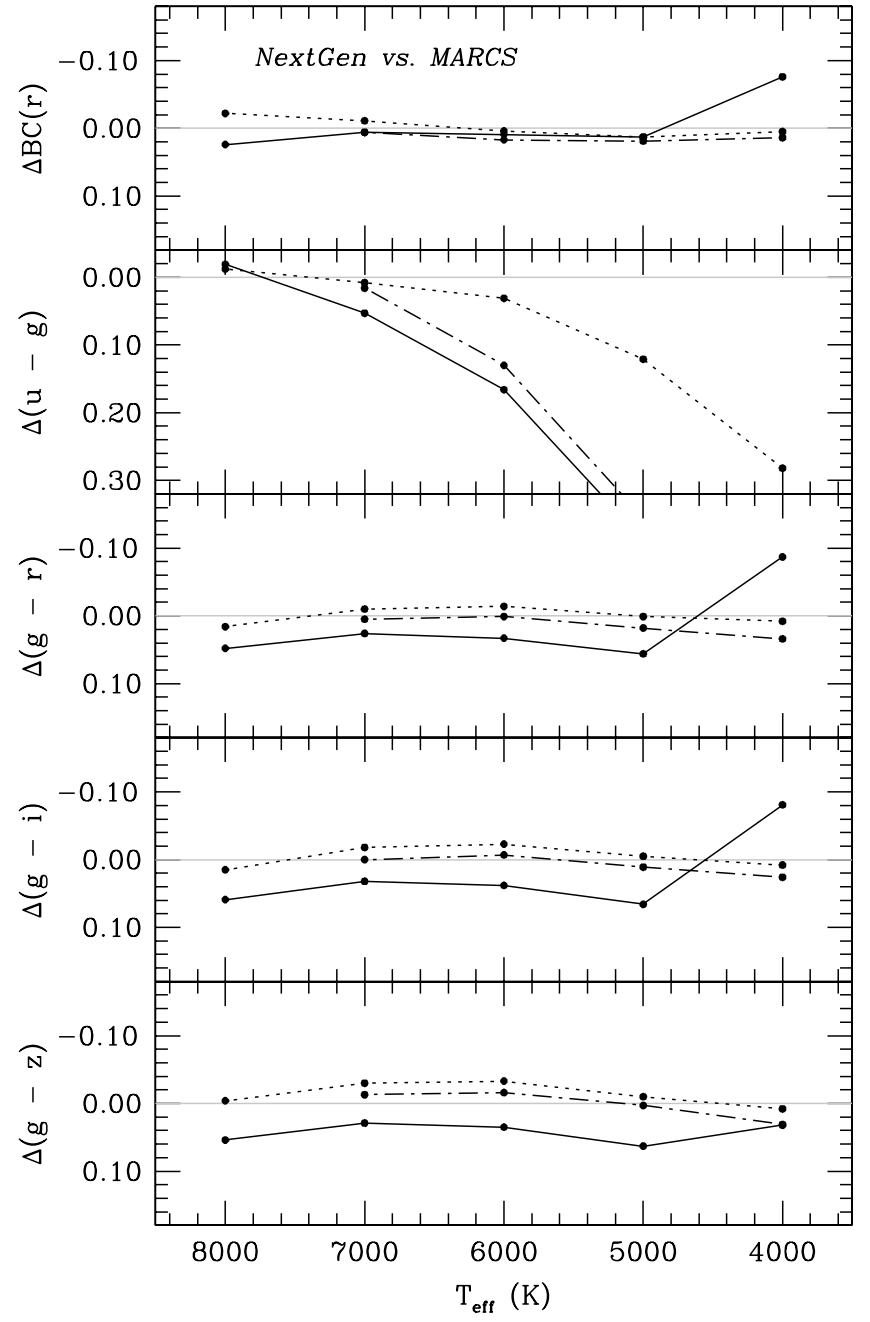

Figure 4. Comparisons in $r$-band bolometric corrections and synthetic colors between PHOENIX/NextGen and MARCS atmosphere models, in the sense of PHOENIX/NextGen minus MARCS. Comparisons are shown at $\log g=4.0$, with three different metallicities: $[\mathrm{Fe} / \mathrm{H}]=-2.0$ (dotted line), -1.0 (dot-dashed line), and +0.0 (solid line). All models assume the solar abundance ratio.

based NextGen grid generated with the PHOENIX code. ${ }^{17}$ Comparisons are shown at $\log g=4.0$ with three different metallicities: $[\mathrm{Fe} / \mathrm{H}]=-2.0$ (dotted line), -1.0 (dot-dashed line), and +0.0 (solid line). However, $\alpha$-element enhanced models were not included in this comparison, because they were not available in the public PHOENIX database. The differences are in the sense of PHOENIX/NextGen minus MARCS. Unlike ATLAS9 model colors, PHOENIX/NextGen models have larger color differences from MARCS in all color indices, especially in $u-g$.

\section{MODEL COMPARISON}

In this section, we assess the accuracy of theoretical isochrones using cluster fiducial sequences for our sample clusters. For our base case, we use YREC+MARCS models to check whether stellar models satisfactorily reproduce observed colors and magnitudes of stars over a wide range of metal abundance. In this comparison, we take into account various systematic errors, such as those from the photometric calibration, cluster distance, and the metallicity scale for globular clusters. We also

\footnotetext{
17 http://phoenix.ens-lyon.fr/Grids/NextGen/SPECTRA/
}

test Padova+ATLAS9 and DSEP+PHOENIX isochrones with cluster fiducial sequences.

\subsection{Systematic Errors in Color-T $T_{\text {eff }}$ Relations}

Figures 5-8 show comparisons between YREC+MARCS models and fiducial sequences in $(g-r, r),(g-i, r),(g-z, r)$, and $(u-g, r)$, respectively. In each panel, fiducial sequences are displayed as either filled or open circles, and the models are shown as solid lines with $[\mathrm{Fe} / \mathrm{H}]$ as indicated on top of each panel. The leftmost panel contains fiducial sequences for the two most metal-poor clusters (M15 and M92). The second and third panels display those for intermediate-metallicity globular clusters (M3, M5, M13, and M71). The last panel displays fiducial sequences for two open clusters (M67 and NGC 6791). To place fiducial sequences on dereddened color versus absolute magnitude diagrams, we adopted the cluster distance and reddening values as discussed in Section 2. The range of $M_{r}$ covered by a fiducial sequence is different from one cluster to the other, because SDSS images were taken in drift-scan or time-delay-and-integrate (TDI) mode, with a fixed effective exposure time of $54.1 \mathrm{~s}$ per source. For the models, we assumed ages of $12.6 \mathrm{Gyr}$ for all globular clusters, $3.5 \mathrm{Gyr}$ for M67, and 10.0 Gyr for NGC 6791. To guide the eye, the approximate positions of the $\operatorname{Sun}^{18}$ are shown on the CMDs as bulls-eyes.

The model comparisons in Figures 5-8 are reproduced in Figures 9-12 as a function of $T_{\text {eff }}$ in $g-r, g-i, g-z$, and $u-g$, respectively. We estimated color differences between models and cluster fiducial sequences at a given $M_{r}$, which was then converted into $T_{\text {eff }}$ in the model. Open circles represent color differences for each of the fiducial points, as shown in Figures 5-8. In this comparison, we used models at the KI03 $[\mathrm{Fe} / \mathrm{H}]$ values for the globular clusters. The dotted lines indicate zero differences between model and the data for each cluster.

In Figures 9-12, the difference between open and filled circles effectively show the size of a systematic error in the model comparison from photometric zero-point errors. In Paper I we used Photo magnitudes in a set of flanking fields, which are far from the crowded cluster field, to set the zero point of DAOPHOT cluster photometry. This procedure was necessary to put DAOPHOT cluster photometry on the Photo magnitude scale. There were typically two SDSS imaging runs covering each cluster, and DAOPHOT photometry was independently calibrated on each run. However, about $1 \%-2 \%$ systematic differences were found in DAOPHOT photometry for overlapping stars in these two runs. For this reason, we combined photometry from two runs by selecting a "reference" run, which was simply chosen based on the sky coverage of a cluster or the run number. Open circles in Figures 9-12 and the data in Figures 5-8 show the case when we adopt these zero points for our sample clusters. On the other hand, filled circles show cases when using an alternate set of zero points for the same stars. In most instances, this is the zero point established by the other run. For M67 and NGC 6791, there were images taken in three SDSS runs, but we only display the case that shows the largest difference in magnitudes. In M71, open circles show the model comparison with the Clem et al. (2008) fiducial sequence (after a photometric transformation to ugriz), where we adopted a 0.01 mag zero-point error in each passband (see below).

The gray strip in Figures 9-12 represents a $\pm 1 \sigma$ range in the color comparison from various systematic errors. We assumed

\footnotetext{
18 See http://www.sdss.org/dr6/algorithms/sdssUBVRITransform.html
} 


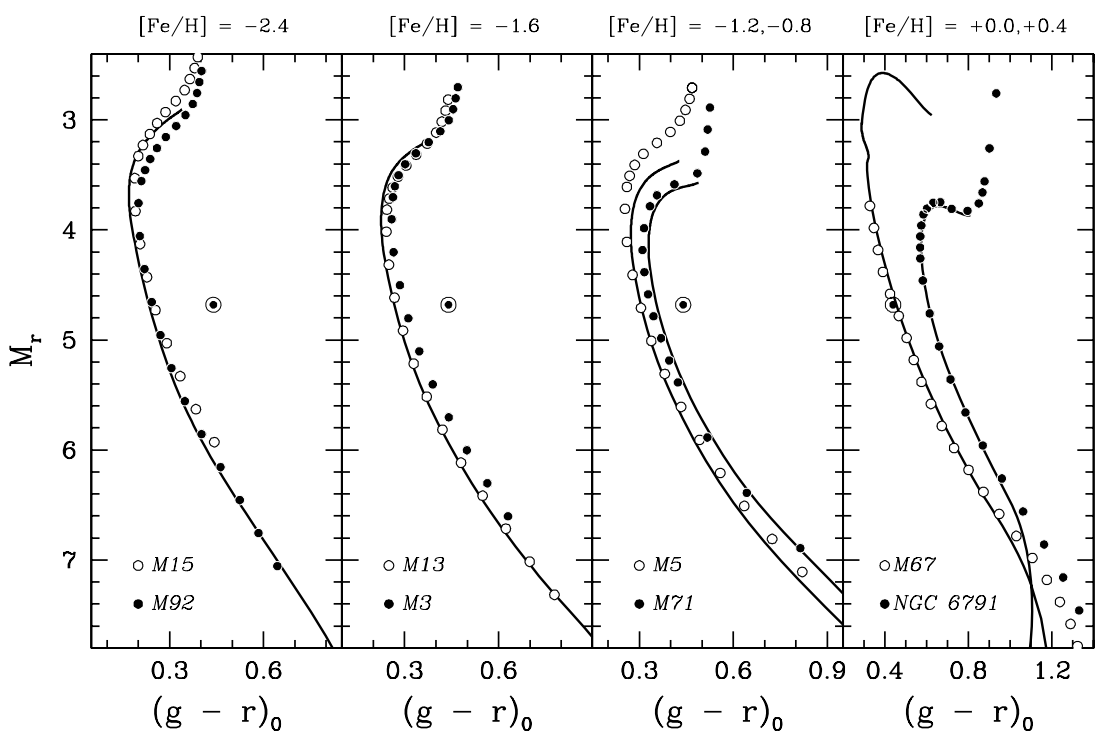

Figure 5. Reddening corrected $\left(g-r, M_{r}\right)$ CMDs for sample clusters. Open and closed circles are fiducial points for each cluster. Left three panels: the solid lines are YREC+MARCS models at the age of $12.6 \mathrm{Gyr}$ with $[\mathrm{Fe} / \mathrm{H}]=-2.4,-1.6,-1.2,-0.8$, as indicated on top of each panel. Distances from the Hipparcos-based subdwarf fitting are assumed, with canonical cluster reddening values (see the text). The fiducial points for M71 are from Clem et al., after transforming $u^{\prime} g^{\prime} r^{\prime} i^{\prime} z^{\prime}$ into ugriz using transformation equations in Tucker et al. Right panel: the solid lines are models with $[\mathrm{Fe} / \mathrm{H}]=+0.0(4.0 \mathrm{Gyr}),+0.3$ (10.0 Gyr). Bull's-eyes mark the approximate position of the Sun, to guide eyes on the metallicity sensitivity of colors and magnitudes.

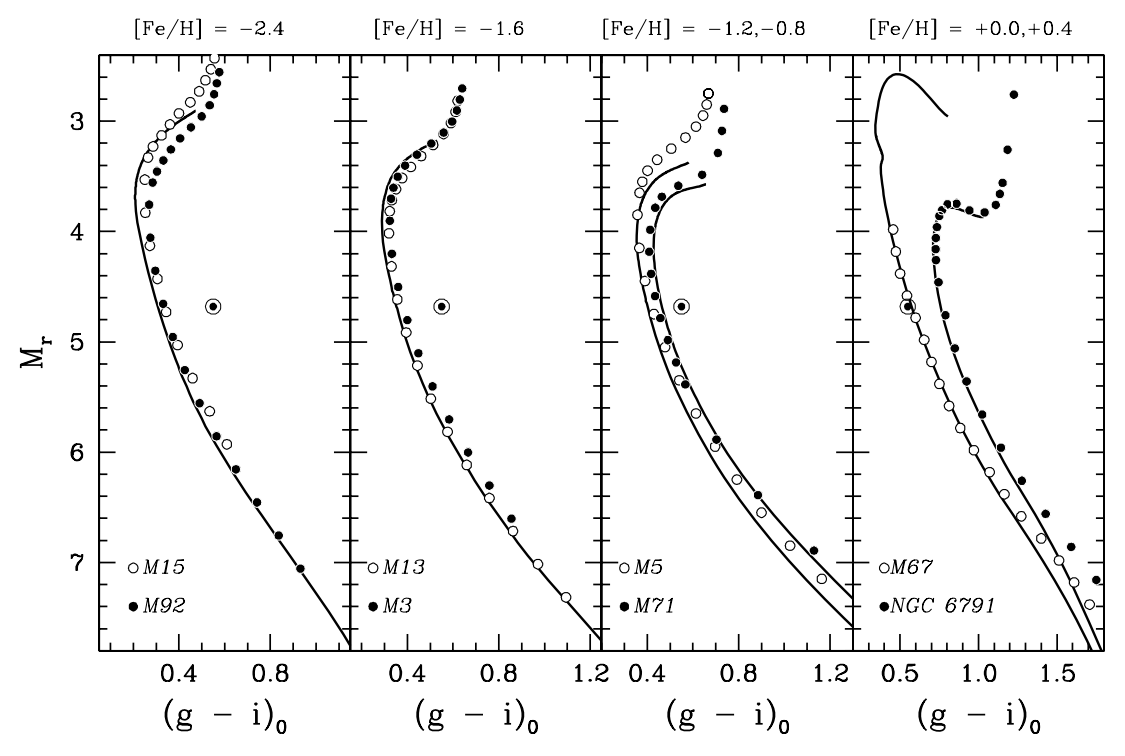

Figure 6. Same as in Figure 5, but $\left(g-i, M_{r}\right)$.

the difference between open and filled circles as a $2 \sigma$ error from the photometric calibration. We adopted quoted errors in distance from individual studies (Section 2). Since KI03 did not provide the distance error, we assumed $\sigma_{(m-M)}=0.15 \mathrm{mag}$, which is a reasonable size for the errors of the Hipparcos-based subdwarf fitting (e.g., Reid 1997, 1998; C00). Note that KI03 derived cluster distances from eyeball fitting on $(B-V, V)$, did not correct for a bias due to binaries (for both the cluster and subdwarf samples), and also did not apply the Lutz-Kelker corrections in their fitting procedure. We assumed $\Delta R_{V}= \pm 0.2$ for the error in the reddening laws, and took a $20 \%$ error in $E(B-V)$ and in the cluster age $(\Delta \log t(\mathrm{Gyr}) \approx 0.1)$. We estimated the error from $[\alpha / \mathrm{Fe}]$ by examining the case of $[\alpha / \mathrm{Fe}]=0.4$ at $[\mathrm{Fe} / \mathrm{H}]<1.0($ Section 3$)$.

Systematic errors in the comparison of model colors are listed in Table 6. They are the average values estimated from all of our sample clusters. To avoid a large discrepancy in the models at the lower MS for M67 and NGC 6791 (see below), we took the average in the color difference over $4500 \leqslant T_{\text {eff }}(\mathrm{K}) \leqslant 6500$ for each cluster. In addition to the individual error sources described above, we also included an error from the fitting residual. Even after shifting isochrones in colors, we often found that they do not perfectly match the shape of the fiducial sequence. We computed this as an error in the average color difference between the model and the cluster fiducial sequence. We discuss the effect of the globular cluster $[\mathrm{Fe} / \mathrm{H}]$ scale in more detail in the next section. The total error is the quadratic sum of all of the error contributions, which is dominated by the error in the cluster distance.

As shown in Figures 9-11, there is no compelling evidence that model colors differ from the observed MS for globular clusters in $g-r, g-i$, and $g-z$. On the other hand, there 


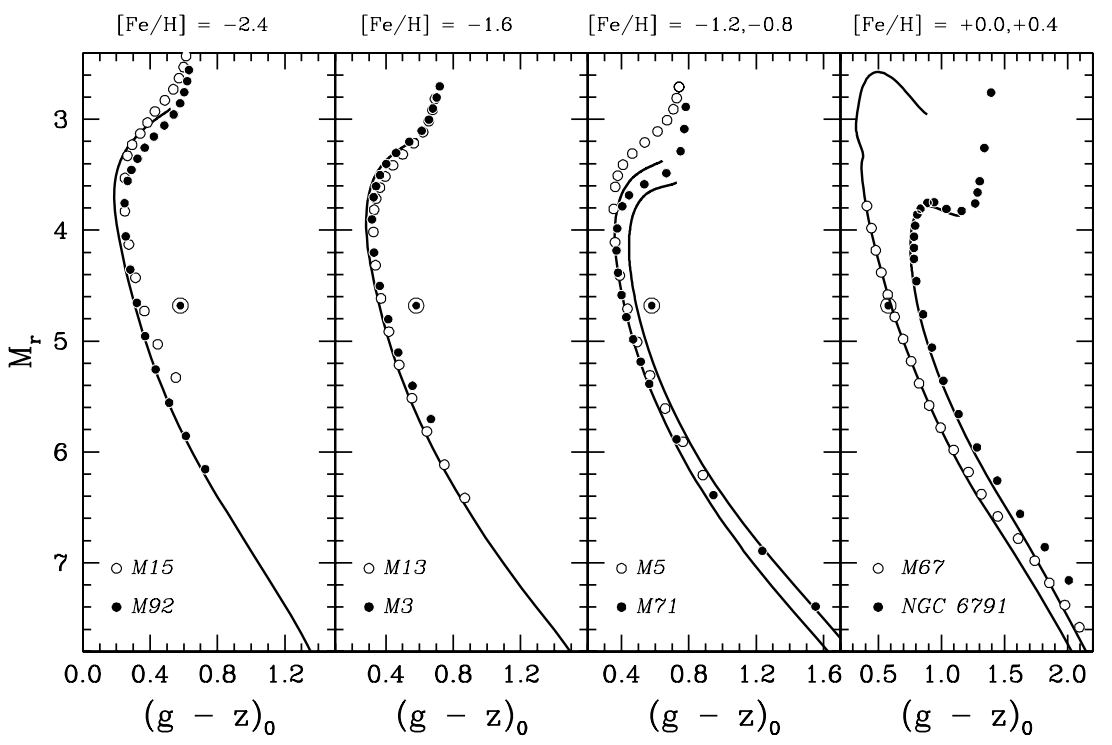

Figure 7. Same as in Figure 5, but $\left(g-z, M_{r}\right)$.

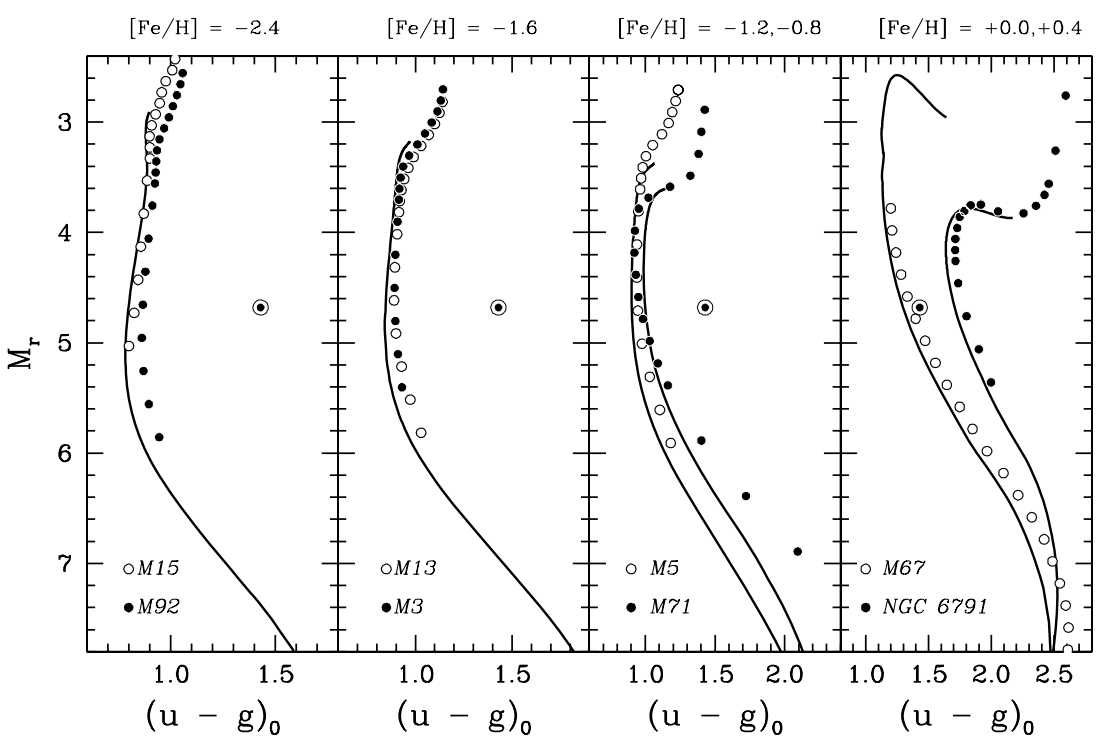

Figure 8. Same as in Figure 5, but $\left(u-g, M_{r}\right)$.

is a strong departure of the models at the lower MS for two open clusters $\left(T_{\text {eff }} \lesssim 4500 \mathrm{~K}\right)$. The models predict bluer colors than the observed ones in all color indices. In fact, the problem has long been noted in other photometric systems, such as the Johnson-Cousins system (e.g., Sandquist 2004), for many contemporary low-mass stellar models. We present empirical corrections for the models in Section 4.4.

There is an $\sim 0.1 \mathrm{mag}$ offset in $u-g$ for all clusters as shown in Figure 12, with no apparent trend in metallicity. Since the other color indices based on the $g$ bandpass exhibit good agreement with the data, the $u-g$ color offset suggests a problem in the $u$ bandpass. The $\sim 5 \%$ color difference is larger than expected from the photometric calibration error in SDSS. Instead, the difference is most likely due to missing opacities in model atmospheres at short wavelengths. The difference is in the sense that models overestimate the flux in the $u$ bandpass. The comparison in $u-g$ for M71 is worse than those for the other clusters in our sample, suggesting a problem in the color transformation for this highly reddened cluster.

\subsection{Metallicity Scale for Globular Clusters}

Figure 13 shows the color differences between YREC+MARCS models and fiducial sequences, as a function of cluster metallicity, on the KI03 scale. Each open circle represents an average color difference for individual clusters over $4500 \leqslant T_{\text {eff }}(\mathrm{K}) \leqslant 6500$. The gray strip displays a $\pm 1 \sigma$ error in Table 6 , excluding the error from $[\mathrm{Fe} / \mathrm{H}]$. As seen in the figure, model colors are generally bluer than fiducial sequences for globular clusters. The average color differences are $\Delta\langle g-r\rangle=+0.008 \pm 0.011, \Delta\langle g-i\rangle=+0.022 \pm 0.017$, $\Delta\langle g-z\rangle=+0.021 \pm 0.018$, and $\Delta\langle u-g\rangle=+0.059 \pm 0.009$ for the five globular clusters in our sample.

Figure 14 shows a model comparison when the CG97 [Fe/H] values are used. In comparison to the KI03 case (Figure 13), color differences between our models and fiducial sequences are reduced by $\sim 0.02$ mag: $\Delta\langle g-r\rangle=-0.006 \pm 0.011$, $\Delta\langle g-i\rangle=+0.004 \pm 0.017, \Delta\langle g-z\rangle=+0.002 \pm 0.018$, and $\Delta\langle u-g\rangle=+0.031 \pm 0.010$. As discussed in $\mathrm{KI} 03$, their $[\mathrm{Fe} / \mathrm{H}]$ 


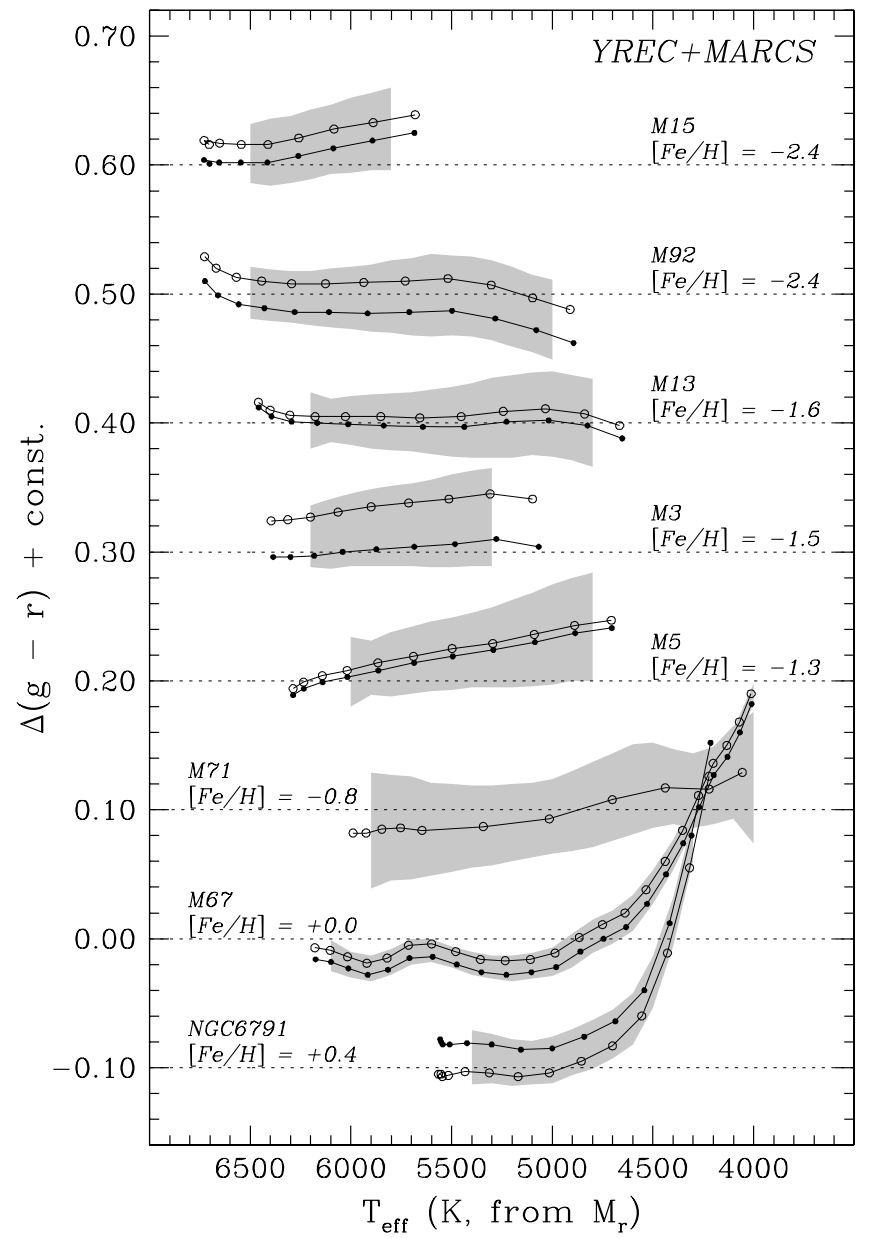

Figure 9. Color differences in $g-r$ between YREC+MARCS models and fiducial sequences. The $\mathrm{KI} 03[\mathrm{Fe} / \mathrm{H}]$ values are assumed with the Hipparcosbased subdwarf-fitting distances for globular clusters. Individual cluster cases are shown in such a way that the most metal-poor cluster is shown at the top, while the most metal-rich open cluster is shown at the bottom, with an offset $\Delta(g-r)=0.10$ between them. The dotted lines indicate zero differences between model and the data. For each cluster, filled and open circles exhibit the size of a systematic error from the photometric calibration (see the text). A gray strip represents $\mathrm{a} \pm 1 \sigma$ range of a total systematic error in the comparison. The result for M71 is based on Clem et al. fiducial sequence (after a photometric transformation to ugriz).

values are about 0.2 dex lower than those from CG97, which make the isochrones redder.

We took the difference from the two $[\mathrm{Fe} / \mathrm{H}]$ scales as an effective $2 \sigma$ error, which is listed in Table 6 . However, the metallicity scale has a relatively small impact on the model comparison, since the total error budget is still dominated by the error in the subdwarf-fitting distances. In the above analyses, we did not take a random error in $[\mathrm{Fe} / \mathrm{H}]\left(\sigma_{\langle[\mathrm{Fe} / \mathrm{H}]\rangle} \approx \pm 0.01-\right.$ $0.02)$ into account, as it is much smaller than the systematic error $\left(\sigma_{\langle[\mathrm{Fe} / \mathrm{H}]\rangle} \approx \pm 0.1\right)$. We note that both CG97 and KI03 were based on spectroscopic measurements of red giants, which could be different from those for dwarfs. However, the difference may not be larger than $\sim 0.1$ dex (e.g., Korn et al. 2006, 2007).

\subsection{Tests of Other Models}

Figure 15 shows a color comparison between cluster fiducial sequences and Padova+ATLAS9 isochrones. Because Girardi et al. (2004) did not include $\alpha$-element enhanced models, we

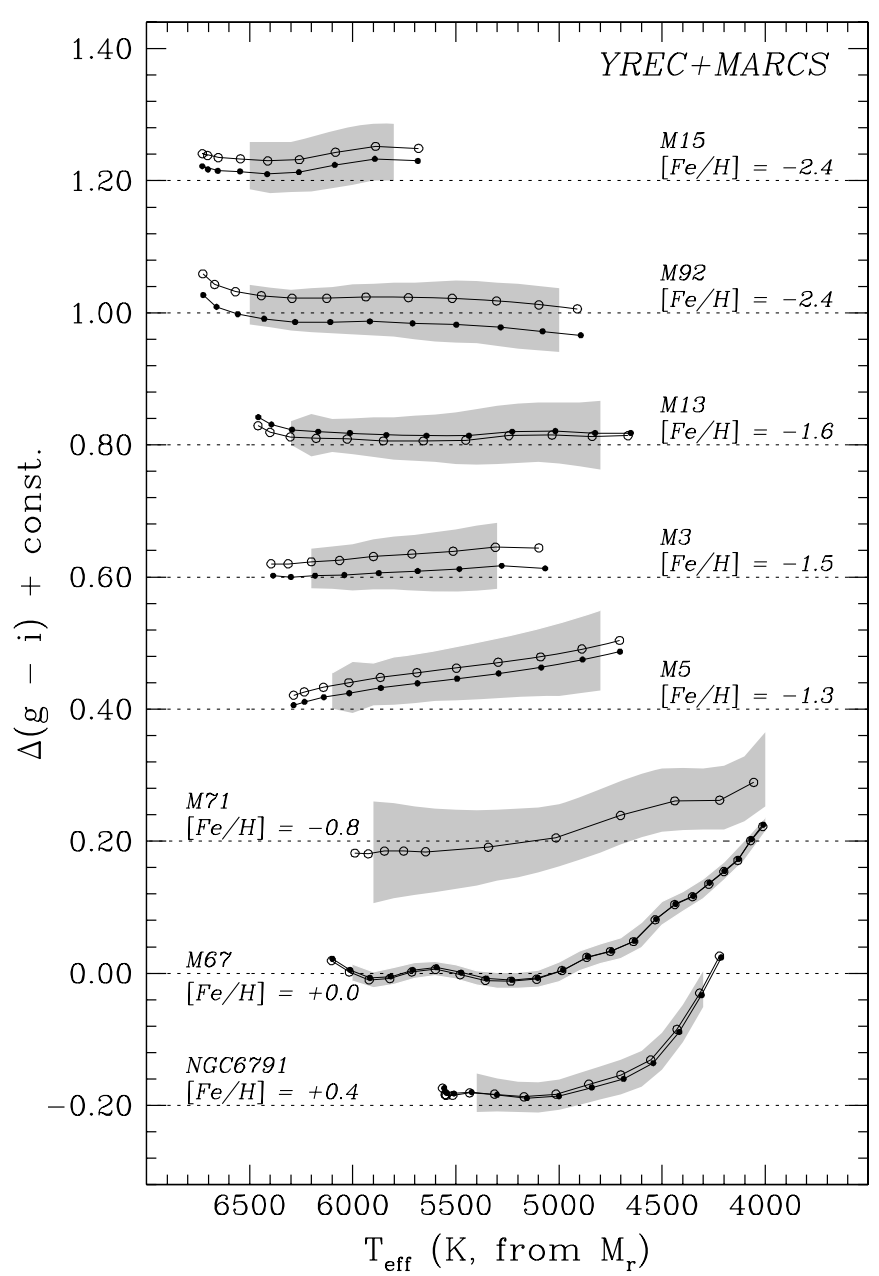

Figure 10. Same as in Figure 9, but model comparisons in $g-i$.

used their models in the comparison at the following $[\mathrm{Fe} / \mathrm{H}]$ values, in order to bracket the observed $[\mathrm{Fe} / \mathrm{H}]$ abundance of a cluster (e.g., Salaris et al. 1993; Kim et al. 2002): $[\mathrm{Fe} / \mathrm{H}]=-2.3$ and -1.7 for $\mathrm{M} 15$ and $\mathrm{M} 92,[\mathrm{Fe} / \mathrm{H}]=-1.7$ and -1.3 for $\mathrm{M} 3$ and $\mathrm{M} 13,[\mathrm{Fe} / \mathrm{H}]=-1.3$ and -0.7 for $\mathrm{M} 5$, and $[\mathrm{Fe} / \mathrm{H}]=$ -0.7 and -0.4 for M71. For the open clusters, we used their models at $[\mathrm{Fe} / \mathrm{H}]=+0.0$ for $\mathrm{M} 67$, and $[\mathrm{Fe} / \mathrm{H}]=+0.37$ for NGC 6791. We applied AB corrections for SDSS magnitudes (Section 3). As shown in Figure 15, Padova+ATLAS9 models describe the observed cluster data reasonably well. However, the error bars (gray strip) are larger than those for the YREC+MARCS cases (Figures 13 and 14), because of the wider range of $[\mathrm{Fe} / \mathrm{H}]$ values adopted in the model comparison.

Similarly, Figure 16 shows a comparison between DSEP+PHOENIX models and fiducial sequences. Since their models are available over a wide range of $[\mathrm{Fe} / \mathrm{H}]$ and $[\alpha / \mathrm{Fe}]$, isochrones were interpolated at the cluster's $[\mathrm{Fe} / \mathrm{H}]$ on the KI03 scale. Although model colors and magnitudes from DSEP+PHOENIX already included AB corrections, 0.01 mag was subtracted from their $g$-band magnitudes, to be consistent with our adopted $\mathrm{AB}$ corrections for SDSS. Although DSEP+PHOENIX models are in reasonably good agreement with the cluster data, they show a large difference in $g-z$ and $u-g$ from the data. The above tests suggest that YREC+MARCS models are generally in better agreement with observed cluster data than these models. 
Table 6

Systematic Errors in the Comparison of Model Colors

\begin{tabular}{|c|c|c|c|c|c|}
\hline Source of Error & $\Delta$ Quantity $^{\mathrm{a}}$ & $\Delta(g-r)$ & $\Delta(g-i)$ & $\Delta(g-z)$ & $\Delta(u-g)$ \\
\hline Fitting residual & 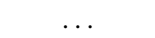 & \pm 0.003 & \pm 0.004 & \pm 0.006 & \pm 0.008 \\
\hline Photometric calibration & $1 \%-2 \%$ & \pm 0.007 & \pm 0.007 & \pm 0.008 & \pm 0.011 \\
\hline$(m-M)_{0}$ & \pm 0.15 & \pm 0.020 & \pm 0.029 & \pm 0.031 & \pm 0.017 \\
\hline$R_{V}$ & \pm 0.2 & \pm 0.002 & \pm 0.002 & \pm 0.001 & \pm 0.006 \\
\hline$E(B-V)$ & $20 \%$ & \pm 0.006 & \pm 0.012 & \pm 0.019 & \pm 0.008 \\
\hline $\log t$ & $20 \%$ & \pm 0.003 & \pm 0.005 & \pm 0.009 & \pm 0.008 \\
\hline$[\alpha / \mathrm{Fe}]^{\mathrm{b}}$ & \pm 0.1 & \pm 0.002 & \pm 0.003 & \pm 0.003 & \pm 0.002 \\
\hline$[\mathrm{Fe} / \mathrm{H}]^{b}$ & \pm 0.1 & \pm 0.007 & \pm 0.009 & \pm 0.009 & \pm 0.014 \\
\hline Total & $\cdots$ & \pm 0.024 & \pm 0.034 & \pm 0.040 & \pm 0.029 \\
\hline
\end{tabular}

Notes. Values are shown for the average color difference over $4500 \leqslant T_{\text {eff }}(\mathrm{K}) \leqslant 6500$.

a Approximate size of the errors. See Section 2 for details.

${ }^{\mathrm{b}}$ Open cluster data are not included in the error estimation.

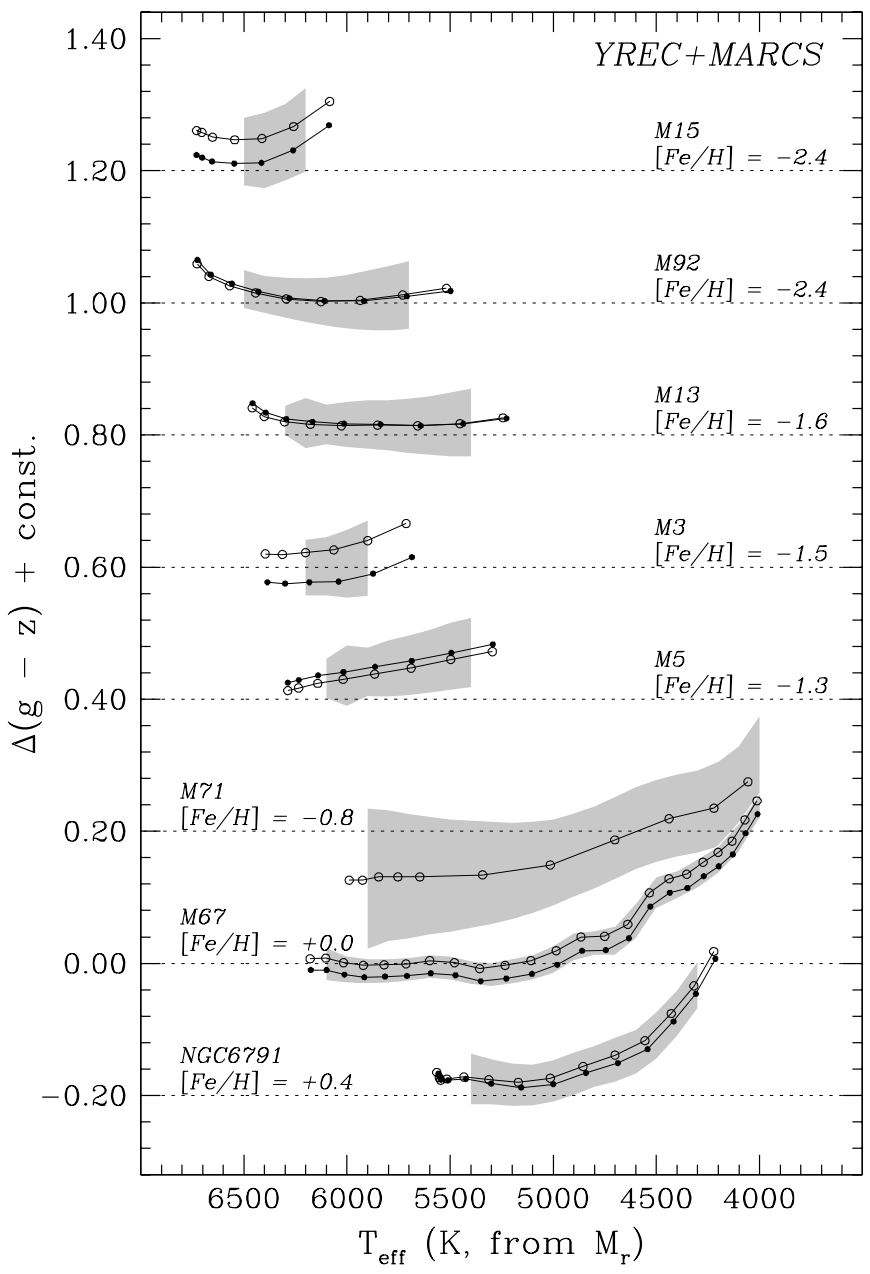

Figure 11. Same as in Figure 9, but model comparisons in $g-z$.

\subsection{Empirical Corrections on Color- $T_{\text {eff }}$ Relations at $[\mathrm{Fe} / \mathrm{H}] \gtrsim \mathrm{O}$}

In the above two sections, we restricted our model comparison to $T_{\text {eff }} \geqslant 4500 \mathrm{~K}$ because the models are in poor agreement with the lower MS at solar and super-solar metallicity (Figures 9-12). Both the Padova+ATLAS9 and DSEP+PHOENIX isochrones show similar discrepancies in colors for open clusters. Since CMDs for globular clusters do not extend far below $T_{\text {eff }} \sim$ $5000 \mathrm{~K}$, similar problems could not be found at the lower MS.

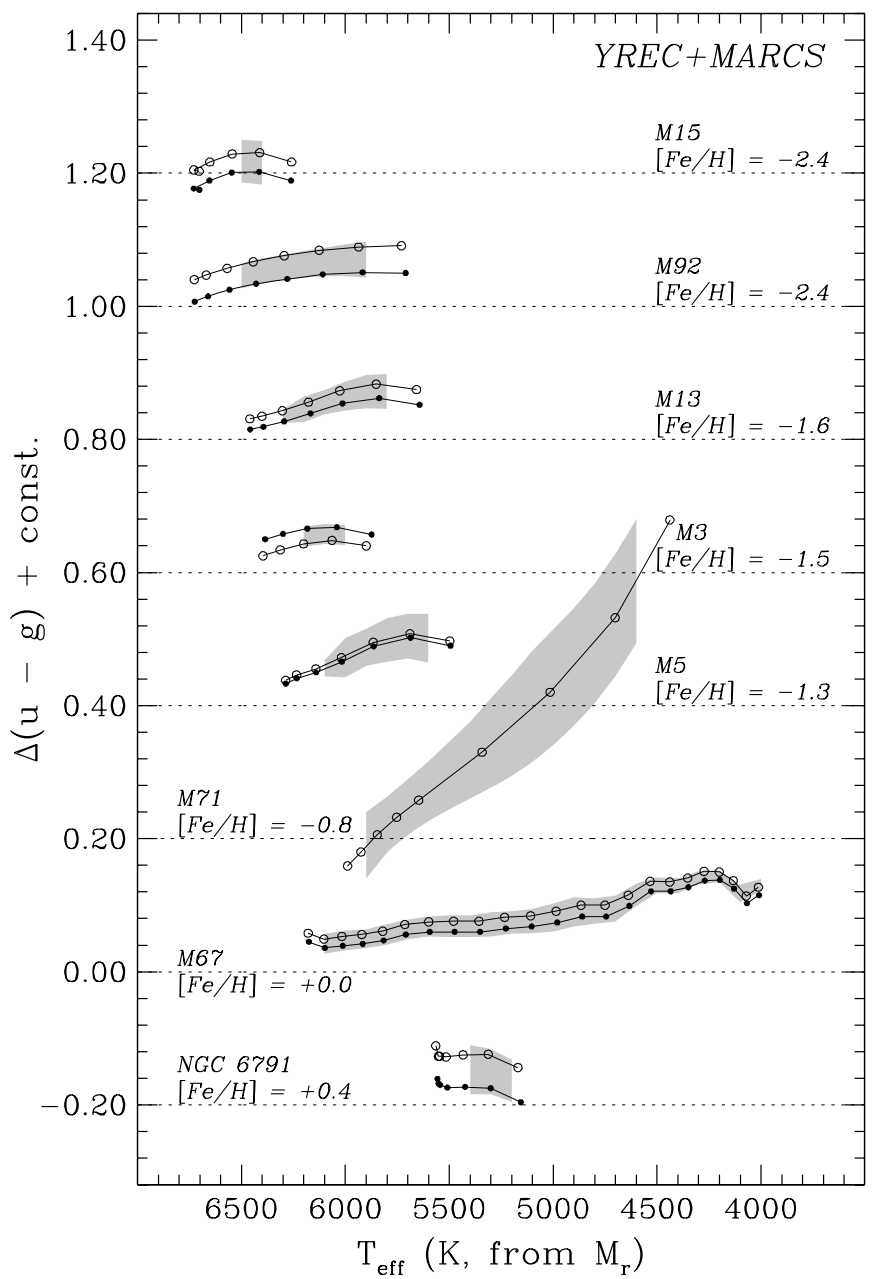

Figure 12. Same as in Figure 9, but model comparisons in $u-g$.

However, the comparison with M71 suggests that model colors and magnitudes are probably accurate at $[\mathrm{Fe} / \mathrm{H}] \lesssim-0.8$

Figure 17 shows color differences for individual cluster members in M67. These stars were selected using photometric filtering of background stars and cluster binaries on $(g-r, r)$, $(g-i, r)$, and $(g-z, r)$, independent from theoretical models (see Paper I). We used a solar metallicity isochrone at an age of 3.5 Gyr. For our calibration exercise in the JohnsonCousins-2MASS filter system (Pinsonneault et al. 2004; An et al. 2007a), we used photometry of the Hyades and Pleiades 


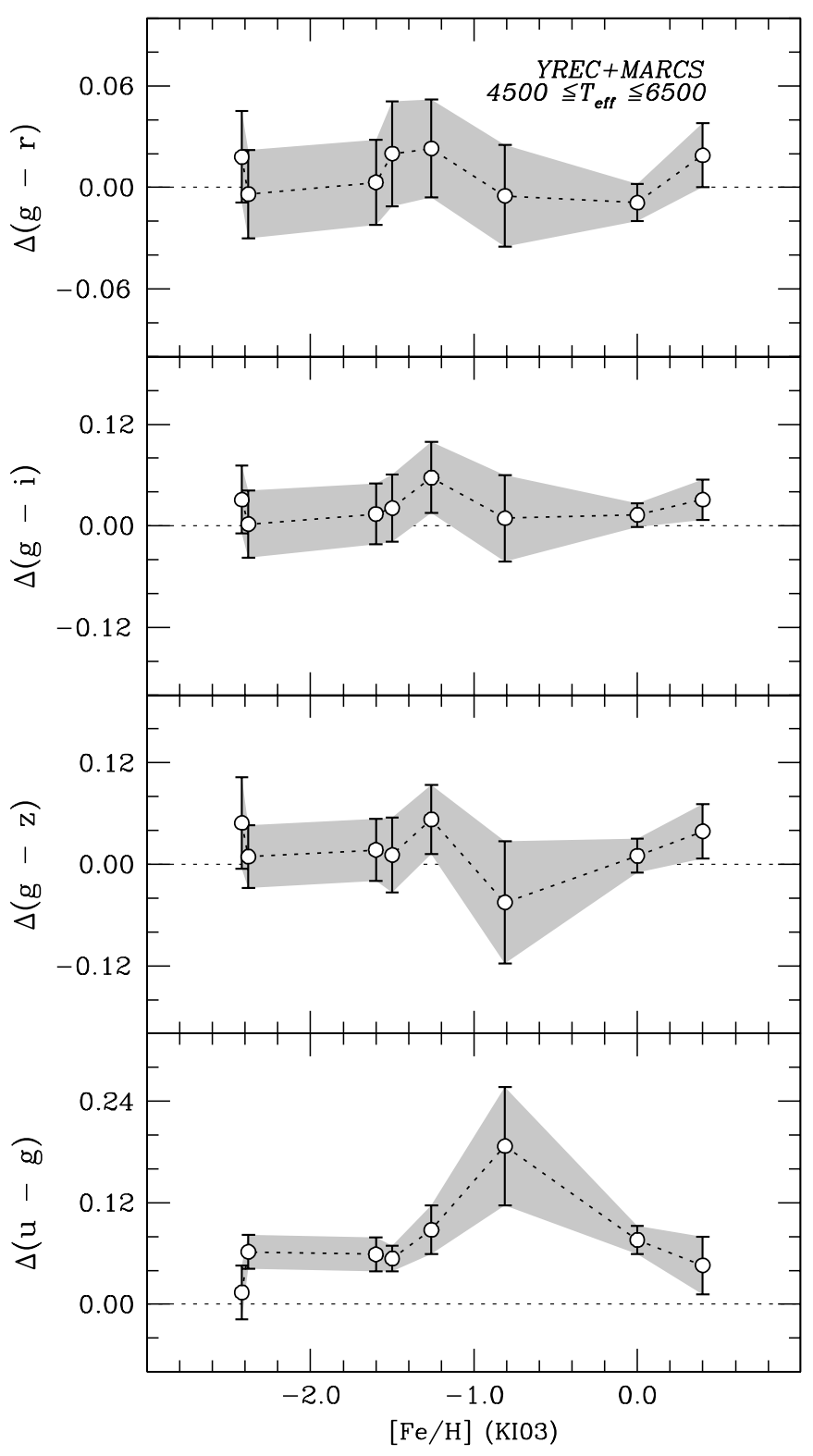

Figure 13. Average color difference between the models and fiducial sequences in ugriz. Open circles represent individual clusters, showing an average color difference computed at $4500 \leqslant T_{\text {eff }}(\mathrm{K}) \leqslant 6500$. For globular clusters the KI03 $[\mathrm{Fe} / \mathrm{H}]$ values are used. The error bars and gray strips represent $\pm 1 \sigma$ range of a total systematic error.

open clusters to define empirical corrections on the color- $T_{\text {eff }}$ relations. Similarly, we could define empirical corrections on the model colors in ugriz based on M67.

The solid lines in Figure 17 are polynomial fits to the points:

$$
\begin{gathered}
\Delta(g-r)=7.613-19.910 \theta+17.279 \theta^{2}-4.983 \theta^{3}, \\
\Delta(g-i)=6.541-16.313 \theta+13.461 \theta^{2}-3.675 \theta^{3}, \\
\Delta(g-z)=3.755-7.595 \theta+4.535 \theta^{2}-0.672 \theta^{3}, \\
\Delta(u-g)=-7.923+24.131 \theta-23.727 \theta^{2}+7.627 \theta^{3},
\end{gathered}
$$

where $\theta \equiv T_{\text {eff }} / 5040 \mathrm{~K}$. These corrections are in the sense that the above values should be added to the model colors, and they are strictly valid at $4000 \leqslant T_{\text {eff }}(\mathrm{K}) \leqslant 6000$.

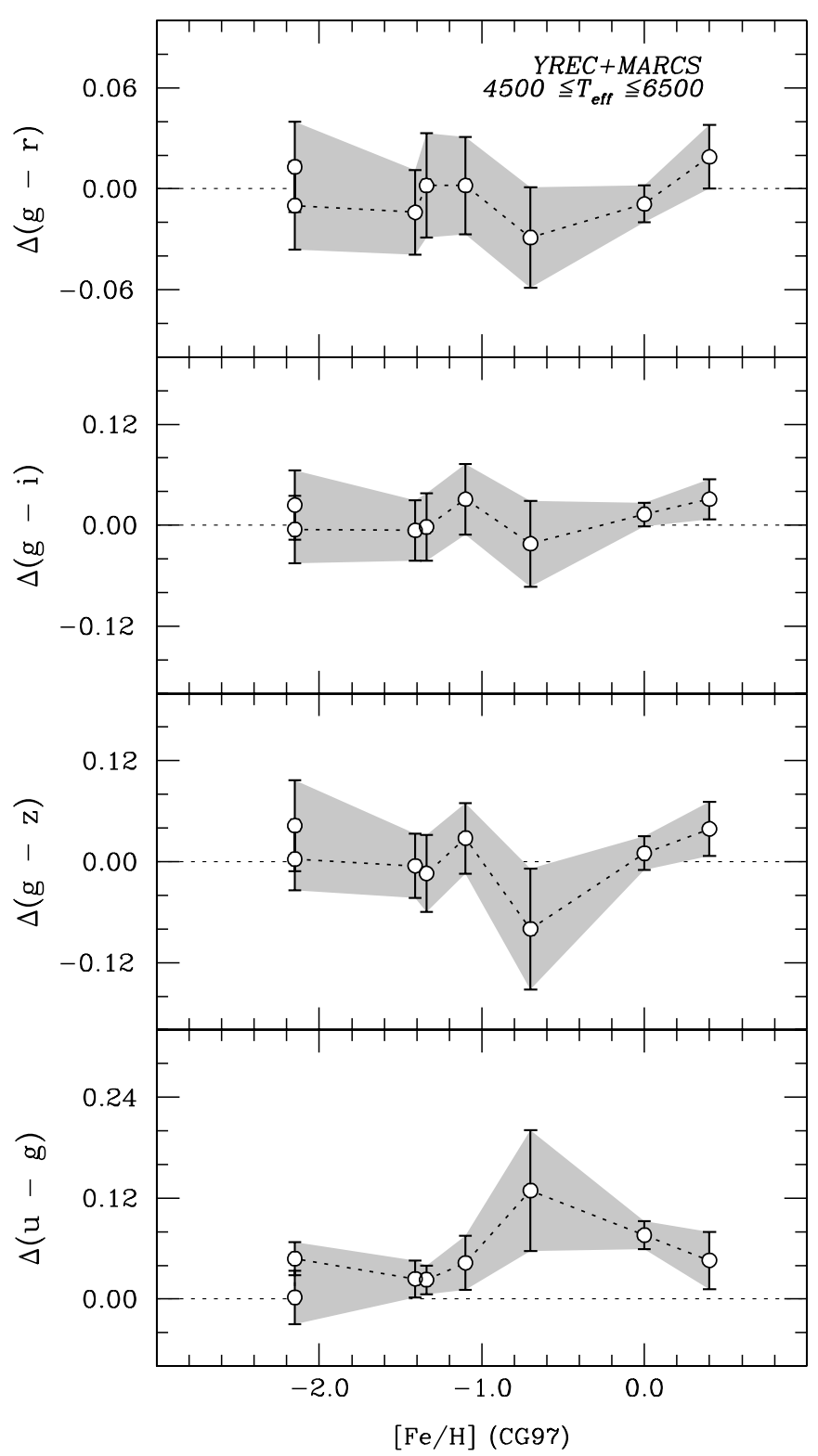

Figure 14. Same as in Figure 13, but when the CG97 [Fe/H] values are used in the model comparison for globular clusters.

It should be noted that the underlying assumptions used in these corrections are somewhat different from our previous work. In Pinsonneault et al. (2003, 2004), the main cause of the residual difference between the model and the observed MS was attributed to the model color- $T_{\text {eff }}$ relations, since the mass-luminosity $-T_{\text {eff }}$ relations were found valid over $4500 \lesssim$ $T_{\text {eff }}(\mathrm{K}) \lesssim 6000$, where the color corrections were defined. On the other hand, the discrepancy between the lower MS and the models at $T_{\text {eff }} \lesssim 4500 \mathrm{~K}$ in Figure 17 could also be due to our limited knowledge of the opacities and/or the equations of state in the models at low temperatures, in addition to a problem in the model atmospheres.

We tested the above corrections using CMDs for NGC 6791, as shown in Figure 18. The dotted lines are YREC+MARCS isochrones without corrections, and the solid lines are those with M67-based color- $T_{\text {eff }}$ corrections. Although [Fe/H]-dependent corrections can be derived using NGC 6791, empirical corrections based on the solar metallicity cluster result in a reasonably good agreement with the observed MS for the super-solar metal- 


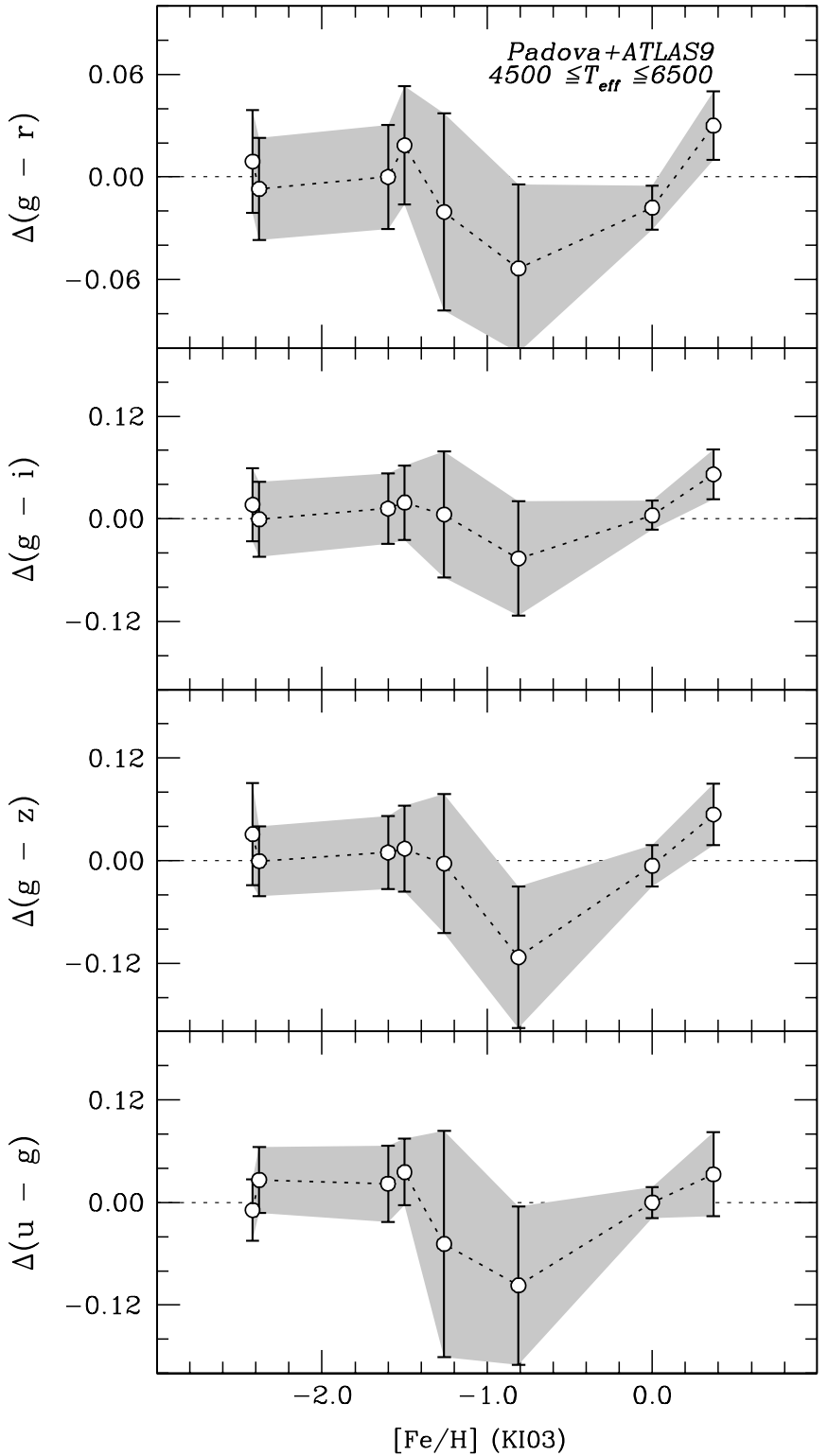

Figure 15. Same as in Figure 13, but for Padova+ATLAS9 models. For each globular cluster, isochrones with two different $[\mathrm{Fe} / \mathrm{H}]$ values are used in the model comparison to bracket the observed cluster abundance (see the text), because Padova+ATLAS9 models do not include $\alpha$-element enhancement.

licity cluster. The calibrated isochrones appear to deviate from the observed MS at the faint end, but the location of the observed MS becomes uncertain as well, due to the increased scatter. Because of a paucity of clusters at $-1 \lesssim[\mathrm{Fe} / \mathrm{H}] \lesssim 0$ in the sample, the lower metallicity limit of the color corrections is not well defined.

\section{DISTANCES AND AGES OF GLOBULAR CLUSTERS}

The ages of Galactic globular clusters have been extensively discussed in the literature, because of their importance in setting a lower limit on the age of the universe, and their implications for the formation of the Galactic halo (e.g., Sandage 1970; Searle \& Zinn 1978; Chaboyer et al. 1998; Rosenberg et al. 1999; VandenBerg 2000; Salaris \& Weiss 2002; De Angeli et al. 2005). Recent age estimates for the Galactic globular clusters are typically found between $\sim 10 \mathrm{Gyr}$ and $\sim 15 \mathrm{Gyr}$ based on various techniques, including MSTO fitting (e.g., Gratton et al.

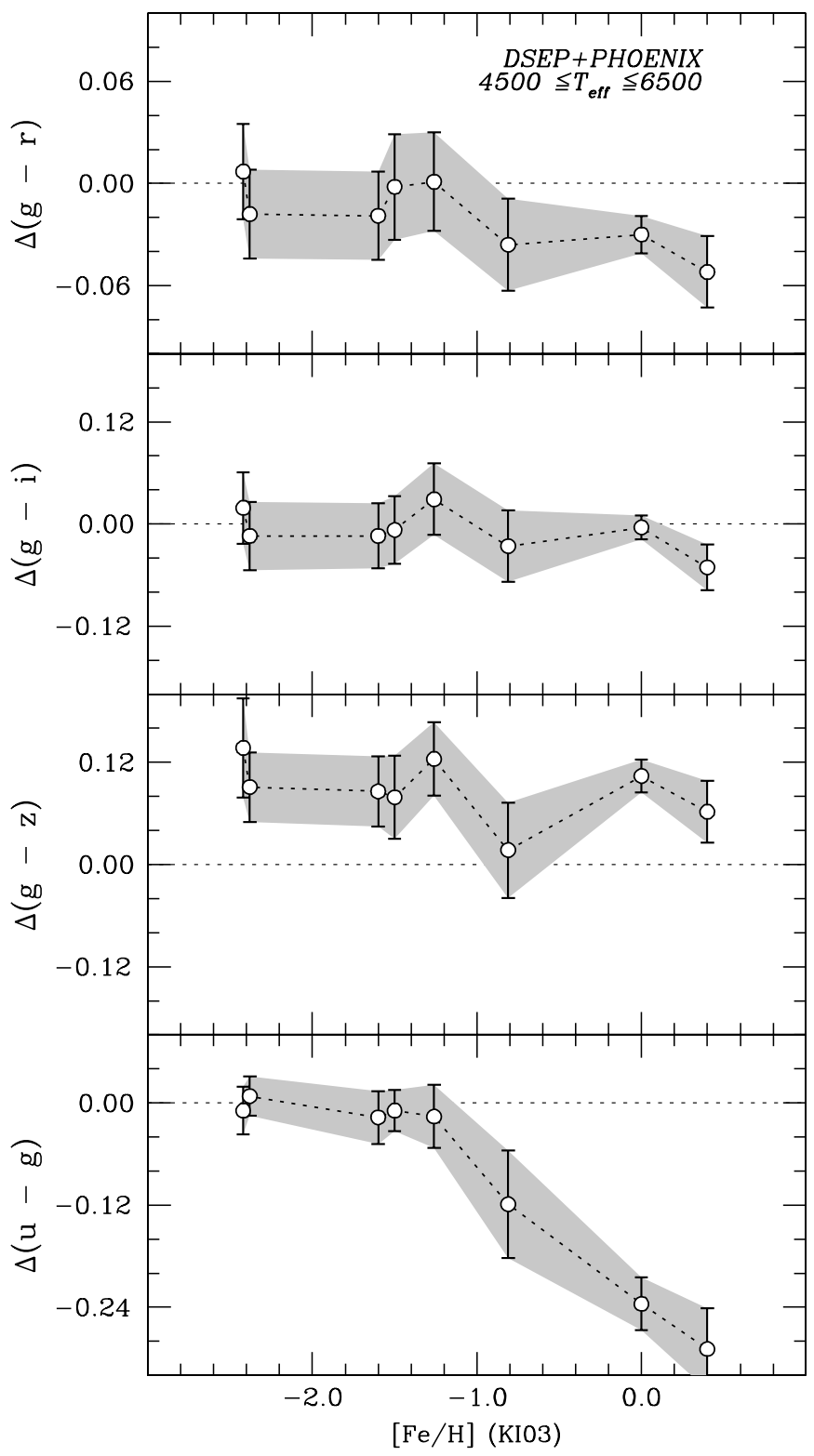

Figure 16. Same as in Figure 13, but for DSEP+PHOENIX models. The KI03 $[\mathrm{Fe} / \mathrm{H}]$ values are used for globular clusters.

1997; Chaboyer et al. 1998; VandenBerg 2000), termination of the white dwarf cooling sequence (e.g., Renzini et al. 1996; Zoccali et al. 2001; Hansen et al. 2002), luminosity function (e.g., Jimenez \& Padoan 1998), and the mass-age relation for an eclipsing binary system (Chaboyer \& Krauss 2002). Although relative age estimates for globular clusters have been well studied (e.g., Stetson et al. 1996; Rosenberg et al. 1999), estimating absolute ages is a more difficult problem, primarily because of the uncertainty in distances to globular clusters (e.g., Vandenberg et al. 1996).

In this section, we estimate distances to our sample globular clusters using the MS-fitting technique without employing empirical color corrections. We then use the derived distance to estimate cluster ages from the MSTO. We test the validity of deriving these quantities from the SDSS photometric database by comparing our values with those in the literature using other methods. In particular, we show that our distances using YREC+MARCS models are in good agreement with the Hipparcos-based subdwarf-fitting distance estimates. 


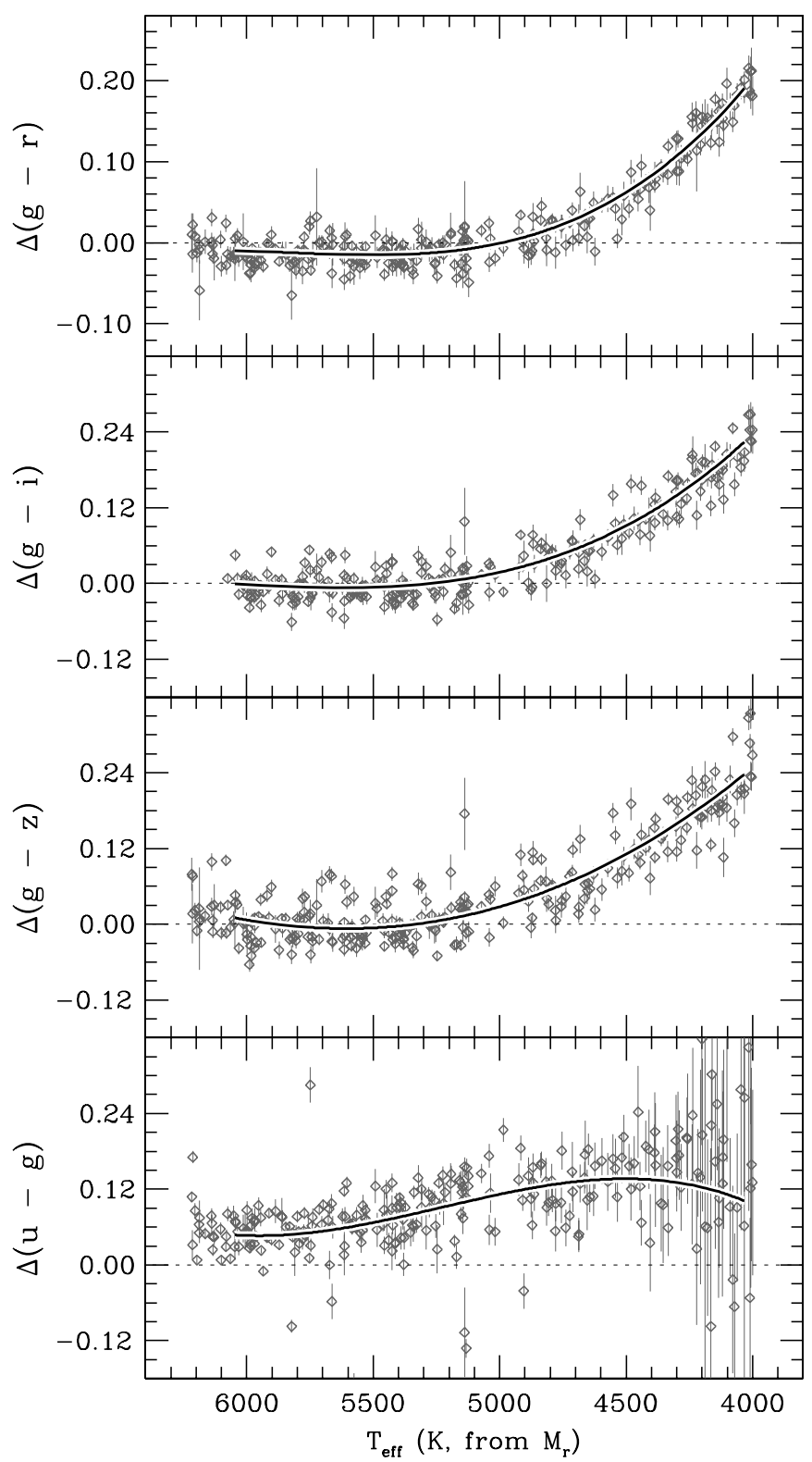

Figure 17. Empirical corrections on theoretical color- $T_{\text {eff }}$ relations. Points represent color differences of individual stars in M67 with respect to a solar metallicity, 3.5 Gyr old YREC+MARCS isochrone. These stars are those selected from a photometric filtering as single MS members based on $(g-r, r)$, $(g-i, r)$, and $(g-z, r)$. The solid lines are empirical color corrections to the models, which are the polynomial fits to the points in each color index.

\subsection{Distances to Globular Clusters}

We obtained distances to globular clusters from CMDs with three color indices $(g-r, g-i$, and $g-z)$. We did not use $u-g$ because of the steep MS slope in the $(u-g, r)$ plane (Figure 8). The utility of the multicolor-fitting technique has been demonstrated in An et al. (2007a, 2007b). With a wide wavelength baseline, stellar properties are not only accurately constrained, but systematic errors can be estimated.

Table 7 lists the derived distance moduli for our sample clusters, using fiducial sequences on $(g-r, r),(g-i, r)$, and $(g-z, r)$. We restricted our fits to $\Delta M_{r} \approx 1$ mag below the MSTO, to minimize any age dependence. For the globular clusters, we list four different sets of distance estimates, on two different metallicity scales, and with two different sets of photometric zero points (Section 4). We computed the size of the error in the average distance modulus from the propagation of a fitting error (shape mismatch) or from a dispersion of distance moduli from three CMDs, whichever is larger (see below for other systematic errors). We found, however, that the latter is always larger than the former by approximately a factor of 4 . The last column lists a standard deviation of the distance moduli from three CMDs, indicating that distances from individual CMDs are consistent with each other to better than $\sim 5 \%$. In Table 7 , we also included results for open clusters (M67 and NGC6791) using purely theoretical models without empirical corrections (Section 4.4) at $r<16.4 \mathrm{mag}$ and $r<21.0 \mathrm{mag}$ for M67 and NGC 6791, respectively.

Table 8 lists systematic errors in distance estimation. We used the same list and size of systematic error sources as in Table 6. An "internal" error was taken from the error in the average distance modulus in Table 7 . Although the adopted size of the photometric calibration errors varies from one cluster to the other, the small size of the errors (e.g., M13) may reflect an accidental agreement of photometry from two SDSS runs. The error from $[\mathrm{Fe} / \mathrm{H}]$ increases at higher metallicities, because the metallicity sensitivity of colors and magnitudes increases steeply with $[\mathrm{Fe} / \mathrm{H}]$. A quadrature sum of all of these systematic errors is generally larger than the internal systematic error. We note that no particular source of systematic errors dominate the total error budget in our distance determination. Average distance moduli for individual clusters and their total errors are listed in the last two columns of Table 2, on the CG97 and KI03 metallicity scales, respectively.

Figure 19 compares our distance moduli for globular clusters with those based on Hipparcos subdwarf fitting. Open circles are those estimated using the $\mathrm{CG} 97[\mathrm{Fe} / \mathrm{H}]$ values, while closed circles are those estimated using the $\mathrm{KIO} 3[\mathrm{Fe} / \mathrm{H}]$ values. Figure 20 shows these differences, as a function of metallicity, on the two different $[\mathrm{Fe} / \mathrm{H}]$ scales. The error bars are the quadrature sum of the errors from both our values and those from the subdwarf-fitting studies. The average difference between these two studies is $\Delta(m-M)_{0}=-0.02 \pm 0.07$ on the CG97 $[\mathrm{Fe} / \mathrm{H}]$ scale, and $\Delta(m-M)_{0}=-0.08 \pm 0.07$ on the KI03 scale. In both cases, our distances are consistent with subdwarf-fitting distances. Furthermore, the agreement between subdwarf fitting and this study indicates that our error estimate $\left(\sigma_{(m-M)} \sim 0.03-0.11 \mathrm{mag}\right)$ for individual clusters is reasonable. In fact, the agreement between the two distance determinations restates our earlier conclusion that the YREC+MARCS models are in good agreement with cluster fiducial sequences.

The distance modulus of M71 from Grundahl et al. (2002) is $0.33 \pm 0.17 \mathrm{mag}$ smaller than the value in Reid (1998), although these studies assumed the same $E(B-V) \approx 0.28$. Our best-fitting distance modulus of this cluster, $(m-M)_{0}=$ $12.86 \pm 0.08$ (KI03) or $12.96 \pm 0.08$ (CG97), are in better agreement with the Grundahl et al. value. Our cluster distance modulus is also in agreement with $(m-M)_{0}=12.76 \pm 0.18$ from Geffert \& Maintz (2000). In addition, the average value from the Harris compilation becomes $(m-M)_{0}=12.92$ when we use $E(B-V)=0.28$, rather than his adopted $E(B-V) \approx 0.32$.

C00 paid particular attention to M92, not only because of its importance as one of the most metal-poor Galactic clusters, but also because of the large discrepancies in its reported distance in the literature. Two factors make measuring the cluster's distance difficult. First, there are only a few metal-poor subdwarfs with $[\mathrm{Fe} / \mathrm{H}] \lesssim-2$ in the Hipparcos catalog. Second, the two 
Table 7

MS-Fitting Distances

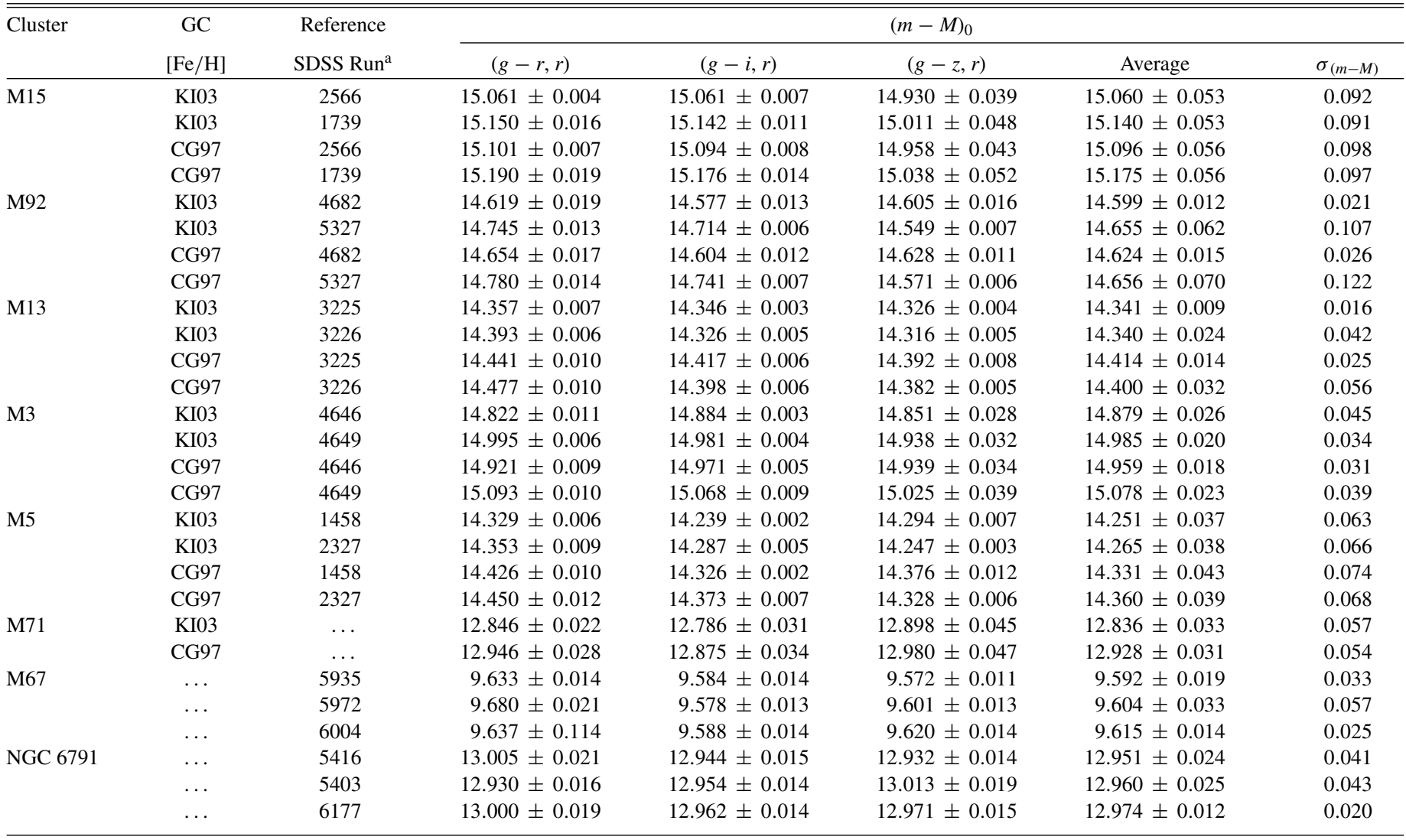

Note. ${ }^{\text {a }}$ SDSS runs selected for the local photometric zero points.

Table 8

Systematic Errors in Distance Moduli for Individual Clusters

\begin{tabular}{|c|c|c|c|c|c|c|c|c|c|}
\hline \multirow{2}{*}{$\begin{array}{l}\text { Source of } \\
\text { Error }\end{array}$} & \multirow[t]{2}{*}{$\Delta$ Quantity } & \multicolumn{8}{|c|}{$\sigma_{(m-M)}$} \\
\hline & & M15 & M92 & M13 & M3 & M5 & M71 & M67 & NGC 6791 \\
\hline Internal & & \pm 0.053 & \pm 0.012 & \pm 0.009 & \pm 0.026 & \pm 0.037 & \pm 0.033 & \pm 0.019 & \pm 0.024 \\
\hline Photometric calibration & $1 \%-2 \%$ & \pm 0.040 & \pm 0.028 & \pm 0.000 & \pm 0.053 & \pm 0.007 & \pm 0.026 & \pm 0.012 & \pm 0.012 \\
\hline$E(B-V)$ & $20 \%$ & \pm 0.089 & \pm 0.013 & \pm 0.043 & \pm 0.008 & \pm 0.019 & \pm 0.055 & \pm 0.010 & \pm 0.032 \\
\hline $\log t$ & $15 \%$ & \pm 0.014 & \pm 0.009 & \pm 0.007 & \pm 0.014 & \pm 0.039 & \pm 0.003 & \pm 0.007 & \pm 0.008 \\
\hline$[\alpha / \mathrm{Fe}]$ & \pm 0.1 & \pm 0.008 & \pm 0.005 & \pm 0.017 & \pm 0.011 & \pm 0.020 & \pm 0.015 & \pm 0.000 & \pm 0.000 \\
\hline
\end{tabular}

Note.

${ }^{a}$ Error in the metallicity scale between CG97 and KI03 for globular clusters. The $\sigma_{[\mathrm{Fe} / \mathrm{H}]}= \pm 0.01$ and $\sigma_{[\mathrm{Fe} / \mathrm{H}]}= \pm 0.03$ were assumed for M67 and NGC 6791 , respectively.

independent photometric studies (Heasley \& Christian 1986; Stetson \& Harris 1988) that provided $B V$ photometry have 0.03 mag differences in colors. As shown in Table 2, with independent photometric data and a wide wavelength coverage in $g r i z$, we found that our distance to M92 is in good agreement with the $\mathrm{C} 00$ distance, independent of the metallicity scale used.

Here, we neglected the effects of unresolved binaries. In An et al. (2007b), we have performed extensive simulations of unresolved binaries in clusters and inspected their influence on the estimation of a MS-fitting distance. After applying photometric filtering, as was done in the present paper for our cluster sample, we found that unresolved binaries can make a
MS look brighter by $\sim 0.007$ mag for a $40 \%$ binary fraction. ${ }^{19}$ The observed binary fraction in globular clusters is typically less than 20\% (e.g., Sollima et al. 2007; Davis et al. 2008, and references therein), which makes the influence of unresolved binaries even smaller.

\subsection{Cluster Ages}

We have derived cluster ages for our sample using MSTO stars, based on the MS-fitting distances to clusters described above. Figures $21-25$ display $(g-r, r),(g-i, r)$, and $(g-z, r)$ CMDs, respectively, for five globular clusters in our sample,

\footnotetext{
19 Binary fraction is defined as the number of binaries divided by the total number of systems.
} 


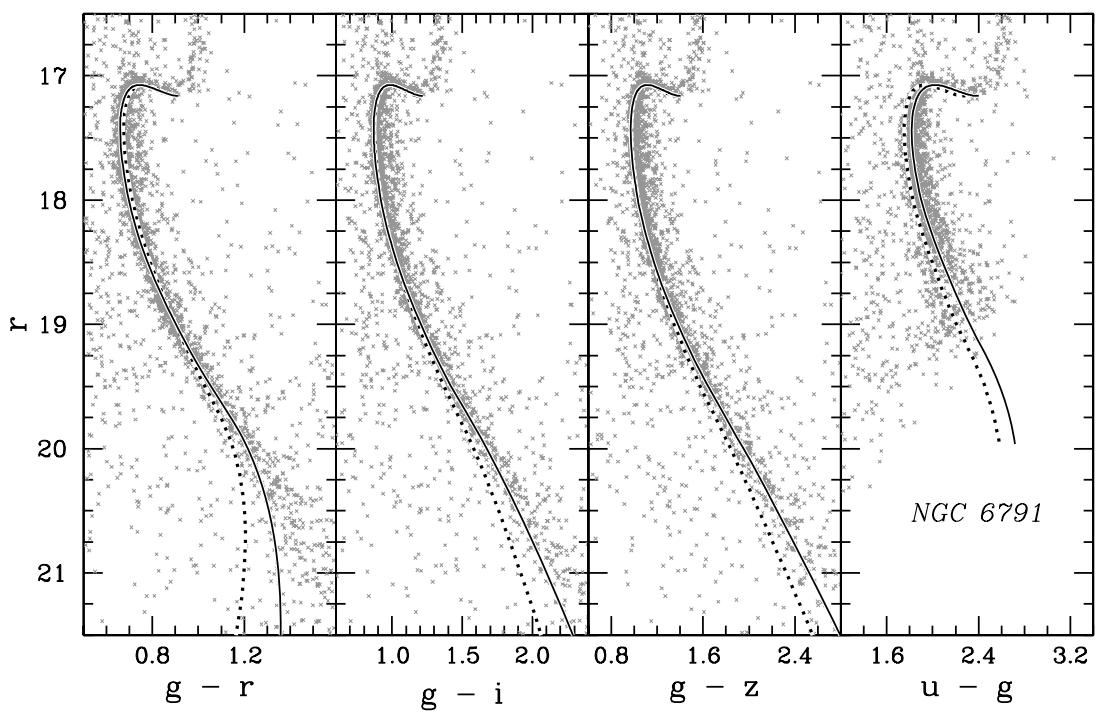

Figure 18. CMDs for NGC 6791. The dotted lines are theoretical models with $[\mathrm{Fe} / \mathrm{H}]=+0.4$ at the age of $10 \mathrm{Gyr}$. The solid lines are models with empirical color $-T_{\text {eff }}$ corrections based on M67 (see Figure 17).

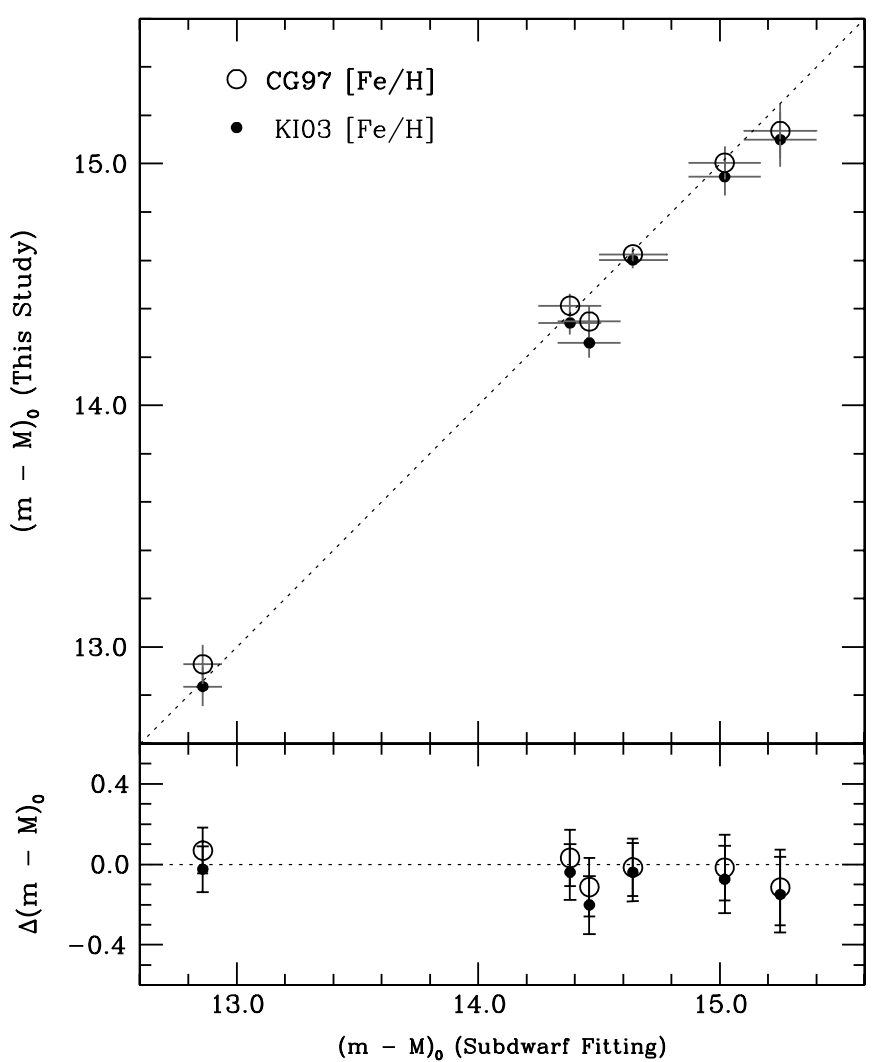

Figure 19. Comparison of distance moduli for globular clusters from the Hipparcos-based subdwarf fitting (see Section 2) and this study. Open circles are those estimated from the $\mathrm{KI} 03[\mathrm{Fe} / \mathrm{H}]$ values, and the closed circles are those from the $\mathrm{CG} 97[\mathrm{Fe} / \mathrm{H}]$ values.

with best-fitting stellar isochrones (solid lines, see below). We fit directly to the CMDs, instead of using the cluster fiducial sequences, because fiducial sequences can be inaccurate near the MSTO (where curvatures are largest on CMDs).

The first panel in Figures 21-25 exhibits residuals on $(g-r, r)$ with respect to a best-fitting model. The points in the remaining three panels are those after applying cuts based on the $\chi$, sharp, and separation indices, as well as the photometric filtering (see Paper I for more details). The photometric filtering

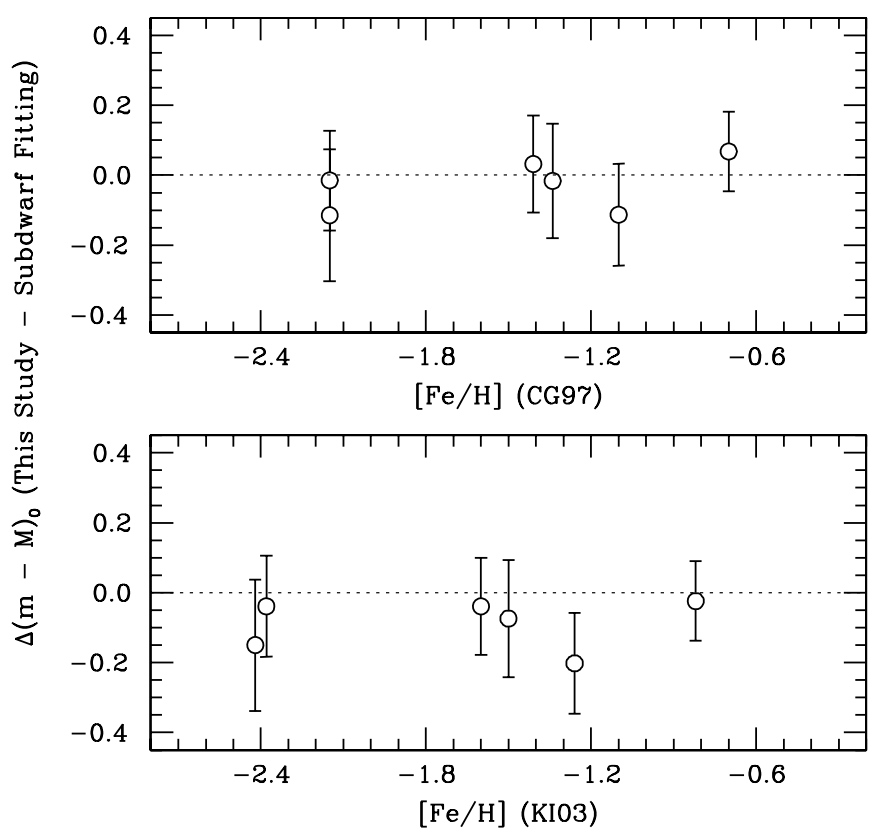

Figure 20. Same as in Figure 19, but as a function of metallicity. Top: comparison of distance moduli when the $\mathrm{CG} 97[\mathrm{Fe} / \mathrm{H}]$ values are adopted in the MS fitting. Bottom: comparison when the $\mathrm{KI} 03[\mathrm{Fe} / \mathrm{H}]$ values are adopted.

routine statistically eliminates cluster non-members and unresolved binaries, independent of the stellar isochrones. However, we note that cuts based on the separation index already make a cluster fiducial sequence sufficiently narrow for well-populated globular clusters such as those in our sample.

To find a best-fitting age on each CMD, we minimized

$$
\chi_{\mathrm{tot}}^{2}=\sum_{i=1}^{N} \frac{\left(X_{i}-X_{m}\right)^{2}}{\sigma_{X, i}^{2}+\left(\gamma \sigma_{r, i}\right)^{2}},
$$

at the best-fitting distance derived simultaneously using MS stars. Here, $X_{i}$ and $\sigma_{X, i}$ are a color and its error for the $i$ th point, respectively. The $\sigma_{r, i}$ is an error in $r$-band magnitude, weighted by the inverse slope of the isochrone $\gamma$. The $X_{m}$ is a model color at the star's $r$-band magnitude. We used stars that are within $\sim 0.5 \mathrm{mag}$ in $r$ on the bright side of the bluest point of the MSTO 


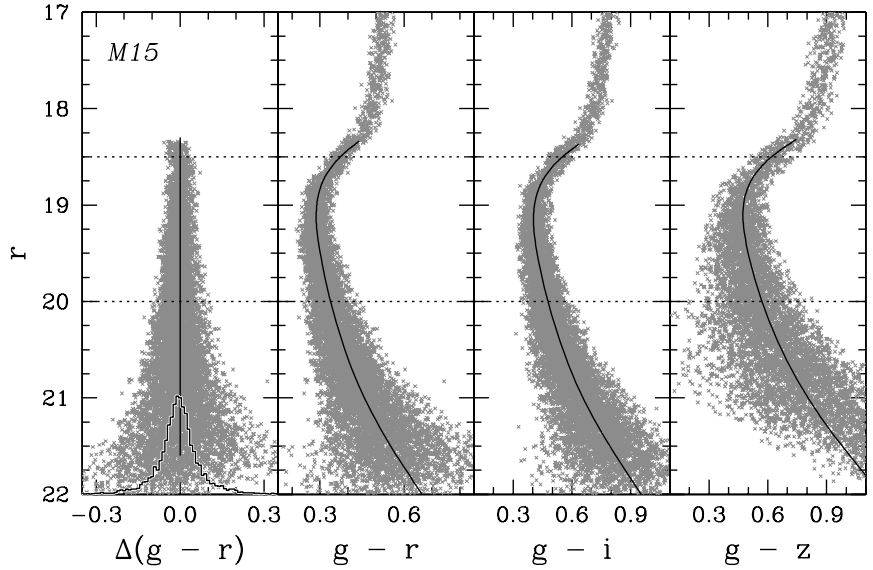

Figure 21. CMDs for M15 with YREC+MARCS models at the best-fitting distance and age (solid line). Models without empirical color corrections are used. The dotted, horizontal lines represent a magnitude range where the isochrone fit was performed to estimate the cluster age. Points are stars that were remained after the photometric filtering (see the text). The leftmost panel shows the fitting residuals in $(g-r, r)$. Histograms are the number distribution of the residuals, with an arbitrary scale on the vertical axis.

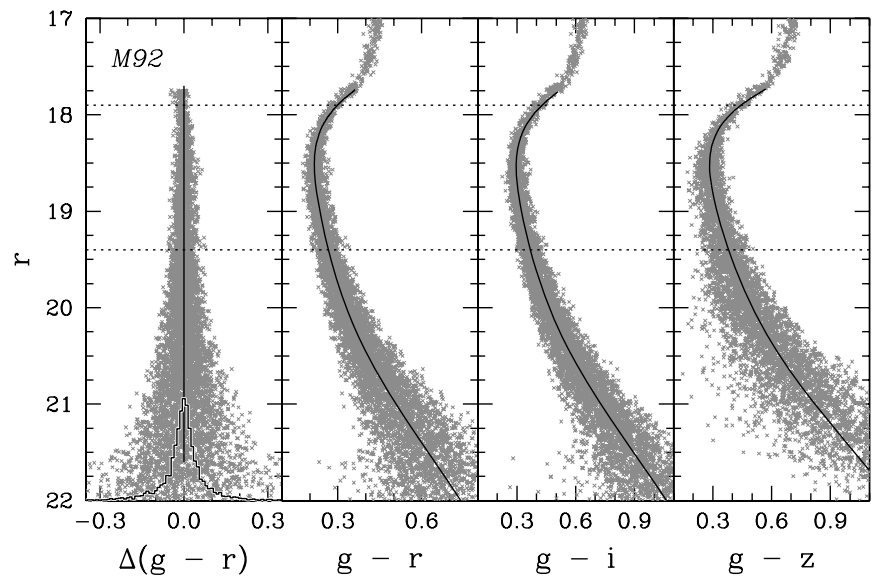

Figure 22. Same as in Figure 21, but for M92.

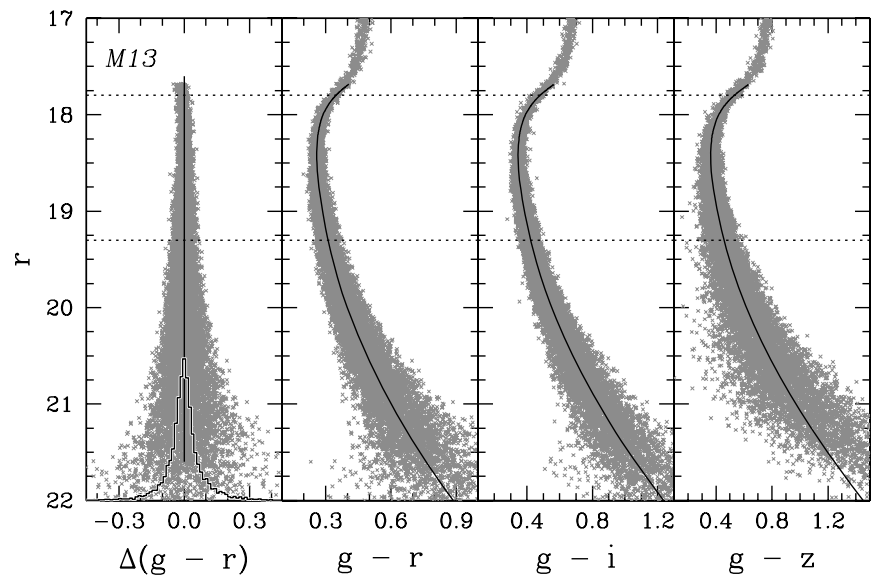

Figure 23. Same as in Figure 21, but for M13.

and $\sim 1.0$ mag on the faint side. The bright magnitude limit was set by the fact that our evolutionary model computations do not reach as far beyond the hydrogen exhaustion in the core. On the red side of subgiant branch (SGB), assumptions on the mixing length and/or the model color- $T_{\text {eff }}$ relations become uncertain as well. The faint end was set at this level, since the MS is

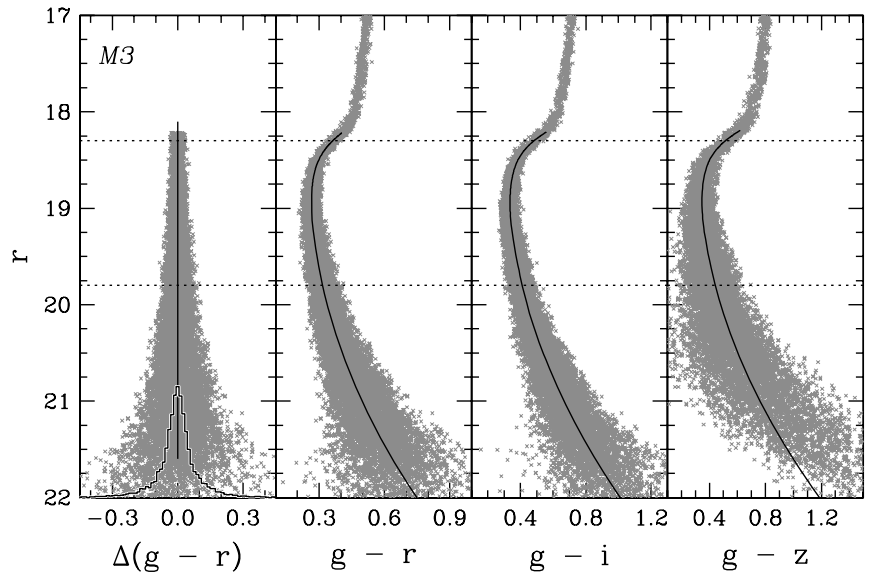

Figure 24. Same as in Figure 21, but for M3.

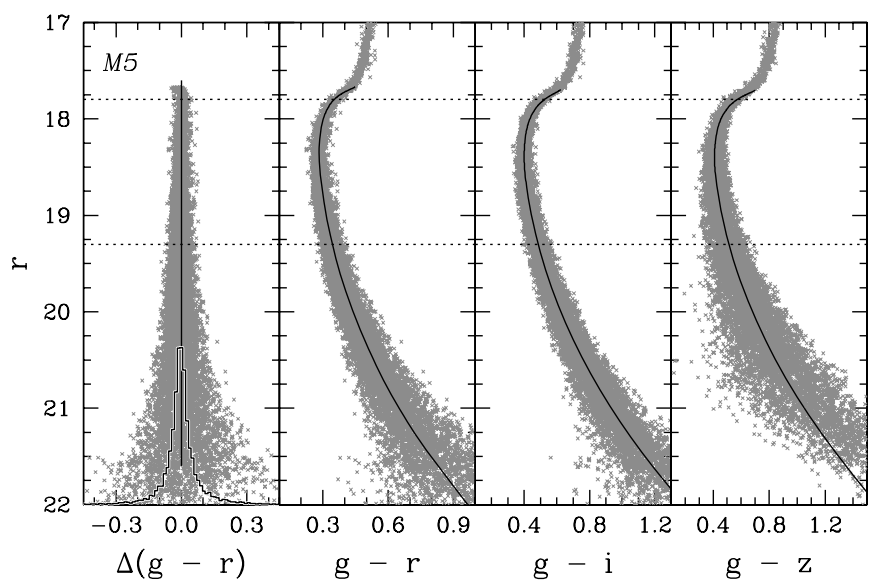

Figure 25. Same as in Figure 21, but for M5.

insensitive to the age below this limit. The dotted horizontal lines in Figures 21-25 show these fitting ranges.

The solid lines in Figures 21-25 represent our best-fitting models for individual CMDs, using $\mathrm{KI} 03[\mathrm{Fe} / \mathrm{H}]$ values. The $g-r$ residuals in the first panels show that fits are excellent from the lower MS to the MSTO. Histograms are also shown to visualize a peaked distribution of color residuals from the model. Reduced $\chi^{2}$ values were between $\sim 0.9$ and $\sim 1.2$ for approximately 2000 stars on each CMD.

Our best-fitting ages are tabulated in Table 9. The errors are the quadrature sums of various systematic errors in the estimation, as listed in Table 10. The same sizes of systematic errors were adopted as in Tables 6 and 8 . The first row in Table 10 shows the scatter in the age estimation from three CMDs $(g-r$, $g-i$, and $g-z$ ), which describes an internal systematic error of $\sim 0.5$ Gyr. Since the distance and age estimates are correlated with each other, we evaluated the effects of various systematic errors, while simultaneously solving for both quantities. In Table 10, we separately tabulated the contribution from an internal error component in distance (see Table 8). We took the difference in age estimates from the CG97 and KI03 $[\mathrm{Fe} / \mathrm{H}]$ scales as a $2 \sigma$ error. Our error estimates indicate that we can obtain cluster ages with an accuracy of $\sim 10 \%-15 \%$ using multiple color indices in SDSS.

The accuracy of our age estimates can be further checked through a comparison with previous studies. Figure 26 shows comparisons of our age estimates with those in two recent studies: VandenBerg (2000, top) and Salaris \& Weiss (2002, 
Table 9

MSTO Ages of Globular Clusters

\begin{tabular}{llc}
\hline \hline Cluster & \multicolumn{2}{c}{ Age $(\mathrm{Gyr})$} \\
\cline { 2 - 3 } & {$[\mathrm{Fe} / \mathrm{H}]_{\mathrm{KI} 03}$} & {$[\mathrm{Fe} / \mathrm{H}]_{\mathrm{CG} 97}$} \\
\hline M15 & $13.9 \pm 2.5$ & $12.8 \pm 2.3$ \\
M92 & $14.4 \pm 0.9$ & $13.5 \pm 0.9$ \\
M13 & $14.3 \pm 1.1$ & $12.3 \pm 1.0$ \\
M3 & $13.3 \pm 1.4$ & $11.4 \pm 1.1$ \\
M5 & $12.2 \pm 1.3$ & $10.5 \pm 1.2$ \\
\hline
\end{tabular}

bottom). VandenBerg (2000) estimated cluster ages from the luminosity difference between his theoretical HB models and two points on the cluster's MS and SGB, which are $0.05 \mathrm{mag}$ redder in $B-V$ than the bluest point of the MSTO. Because the cluster $[\mathrm{Fe} / \mathrm{H}]$ values adopted in VandenBerg (2000) were generally found to lie between the CG97 or KI03 values, ${ }^{20}$ we used the average of our age estimates at the CG97 and $\mathrm{KI} 03[\mathrm{Fe} / \mathrm{H}]$ values in the comparison. On the other hand, Salaris \& Weiss (2002) derived ages for M3 and M15 from the $V$ magnitude difference between zero-age HB and MSTO, but employed relative age estimates for other clusters in our sample from the color difference in $B-V$ or $V-I_{C}$ between the MSTO and the base of RGB. They presented ages from both the Zinn \& West (1984) and CG97 [Fe/H] scales, but only those from the latter $[\mathrm{Fe} / \mathrm{H}]$ scale are shown in Figure 26.

There is excellent agreement between our age estimates and those in VandenBerg (2000; Figure 26), even though they employed independent photometry, photometric filters, stellar isochrones, and different methods of estimating cluster distances and ages. In addition to the above two studies, C00 derived ages for nine globular clusters by comparing MSTO luminosities with model isochrones (Straniero et al. 1997). They obtained 14.8, 12.6, and 11.2 Gyr for M92, M13, and M5, respectively, which are in agreement with our values. The outcome of the comparisons between ours and the preceding studies demonstrates that cluster ages derived with the SDSS ugriz system are reliable, and that our error estimates on age are reasonable.

However, we also noted above that theoretical errors (e.g., Vandenberg et al. 1996; Chaboyer et al. 1998; Krauss \& Chaboyer 2003) were not included in our total error budget. For example, the inclusion of the microscopic diffusion (Korn et al. $2006,2007)$ can systematically reduce stellar ages by $\sim 10 \%$ (e.g., Chaboyer et al. 2001; VandenBerg et al. 2002; Michaud

${ }^{20}$ VandenBerg (2000) adopted $[\mathrm{Fe} / \mathrm{H}]=-2.30$ for M15 and M92, $[\mathrm{Fe} / \mathrm{H}]$ $=-1.60$ for $\mathrm{M} 3$ and $\mathrm{M} 13$, and $[\mathrm{Fe} / \mathrm{H}]=-1.40$ for $\mathrm{M} 5$.

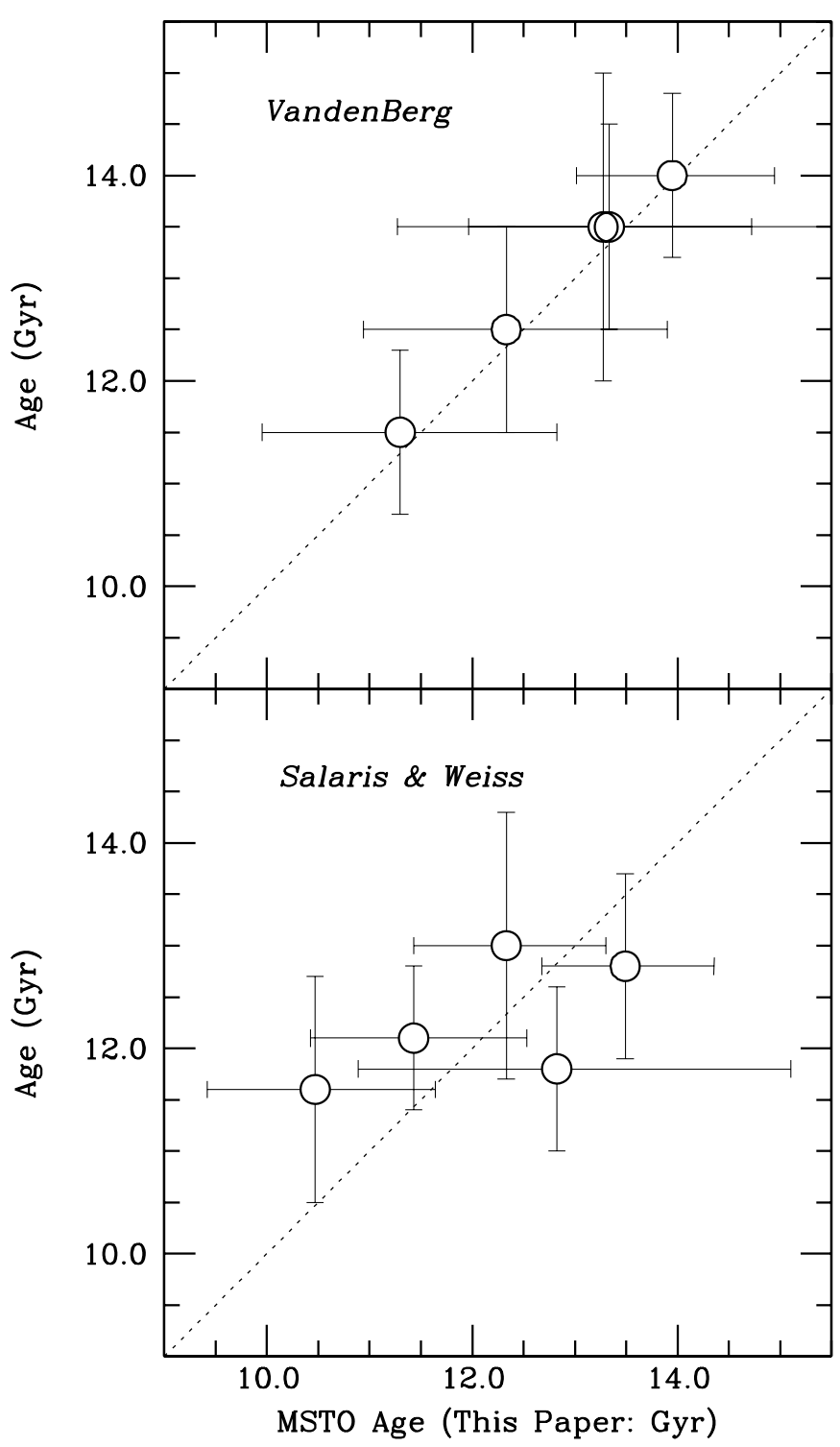

Figure 26. Top: comparison of globular cluster age estimates in VandenBerg (2000) and those in this study. The latter are the average values computed from the CG97 and $\mathrm{KI} 03[\mathrm{Fe} / \mathrm{H}]$ values, while the former age estimates are based on those $[\mathrm{Fe} / \mathrm{H}]$ values adopted in that study (see the text). Bottom: comparison between Salaris \& Weiss (2002) and this study. Both of these age estimates are based on the CG97 [Fe/H] values.

et al. 2004). Nevertheless, our fitting results (Figures 21-25) are encouraging, showing excellent fits in all three of the color

Table 10

Systematic Errors in the Ages of Globular Clusters

\begin{tabular}{|c|c|c|c|c|c|c|}
\hline \multirow{2}{*}{$\begin{array}{l}\text { Source of } \\
\text { Error }\end{array}$} & \multirow[t]{2}{*}{$\Delta$ Quantity } & \multicolumn{5}{|c|}{$\sigma_{\log t}$} \\
\hline & & M15 & M92 & M13 & M3 & M5 \\
\hline Internal & & \pm 0.024 & \pm 0.014 & \pm 0.009 & \pm 0.012 & \pm 0.027 \\
\hline \multirow{2}{*}{$\begin{array}{l}\text { Photometric calibration } \\
(m-M)_{0}\end{array}$} & $1 \%-2 \%$ & \pm 0.022 & \pm 0.015 & \pm 0.006 & \pm 0.024 & \pm 0.004 \\
\hline & Internal & \pm 0.003 & \pm 0.003 & \pm 0.001 & \pm 0.003 & \pm 0.002 \\
\hline$R_{V}$ & \pm 0.2 & \pm 0.009 & \pm 0.003 & \pm 0.002 & \pm 0.000 & \pm 0.002 \\
\hline$E(B-V)$ & $20 \%$ & \pm 0.061 & \pm 0.012 & \pm 0.019 & \pm 0.007 & \pm 0.019 \\
\hline$[\alpha / \mathrm{Fe}]$ & \pm 0.1 & \pm 0.010 & \pm 0.011 & \pm 0.023 & \pm 0.029 & \pm 0.028 \\
\hline$[\mathrm{Fe} / \mathrm{H}]$ & \pm 0.1 & \pm 0.018 & \pm 0.015 & \pm 0.032 & \pm 0.033 & \pm 0.034 \\
\hline \multirow[t]{2}{*}{ Total } & $\ldots$ & \pm 0.073 & \pm 0.030 & \pm 0.045 & \pm 0.052 & \pm 0.055 \\
\hline & & $( \pm 16.8 \%)$ & $( \pm 7.0 \%)$ & $( \pm 10.4 \%)$ & $( \pm 12.0 \%)$ & $( \pm 12.7 \%)$ \\
\hline
\end{tabular}




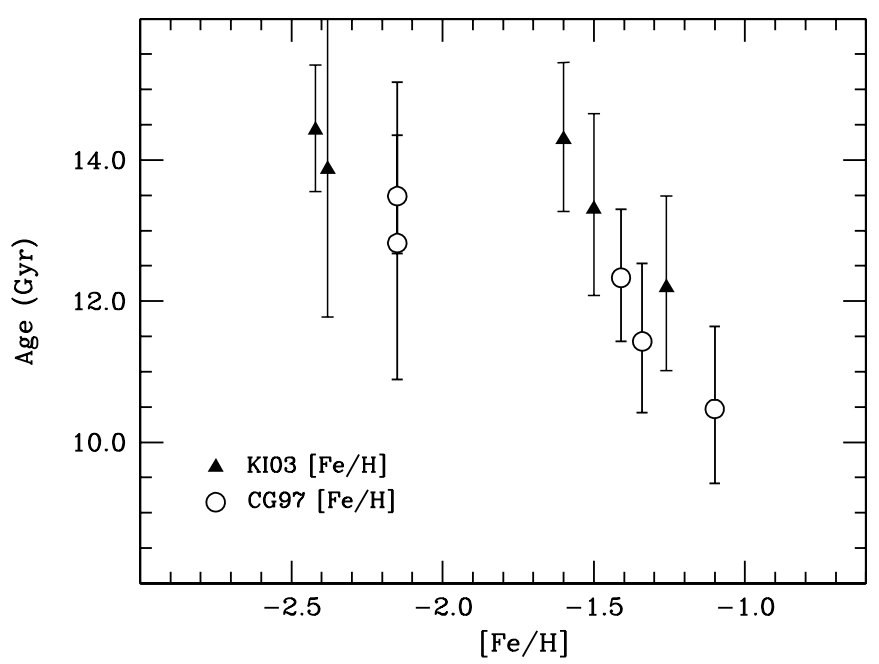

Figure 27. Turnoff ages of globular clusters in this study as a function of metallicity. Filled triangles and open circles represent the cases of adopting the $\mathrm{KI} 03$ or $\mathrm{CG} 97[\mathrm{Fe} / \mathrm{H}]$ values, respectively.

indices. This suggests that our mixing-length calibration based on the Sun is valid for stars in globular clusters, and that theoretical color $-T_{\text {eff }}$ relations are also good for MSTO stars, as we found for MS stars in Section 4.

Cluster ages have traditionally been estimated from the absolute $V$ magnitude of the bluest point on MSTO (e.g., $\Delta V_{\mathrm{TO}}^{\mathrm{HB}}$ method). This was because the luminosity of the MSTO was considered to be the most robustly predicted quantity in stellar evolutionary models (e.g., Bolte \& Hogan 1995; Chaboyer et al. 1996b). Our approach of fitting isochrones to the whole MSTO has been cautioned against in the literature (e.g., Renzini 1991), primarily because of limits on knowledge of the mixinglength parameter and the color- $T_{\text {eff }}$ relations used in the models. However, the $\Delta V_{\mathrm{TO}}^{\mathrm{HB}}$ method has its own limitations, in particular the observational difficulty of pinning down the bluest MSTO point. Because the MS is almost vertical in that region, the magnitude of the MSTO cannot be better determined than $\Delta V \sim 0.1 \mathrm{mag}$, which then produces an error of $\sim 1.5 \mathrm{Gyr}$ in age. Subsequent studies modified the $\Delta V_{\mathrm{TO}}^{\mathrm{HB}}$ technique in such a way that slightly redder points than the MSTO are used as an age indicator (e.g., Chaboyer et al. 1996a; VandenBerg 2000). However, this method implicitly assumes that the shape of MSTO in the models is correct, as we have in this paper.

Figure 27 shows MSTO ages for our sample globular clusters as a function of metallicity. The open circles are those estimated from the $\mathrm{CG} 97[\mathrm{Fe} / \mathrm{H}]$ values, while the filled triangles are those from the KI03 values. The error bars represent the total error in age, as listed in Table 9. With constraints on the distance, we can inspect how stellar ages can be affected by secondary factors, such as the cluster $[\mathrm{Fe} / \mathrm{H}]$ scale. When the $\mathrm{KI} 03[\mathrm{Fe} / \mathrm{H}]$ values are adopted, the average age of our sample clusters is $13.8 \pm 0.5 \mathrm{Gyr}$. Some of the clusters are even older than the inferred age of the universe based on the Wilkinson Microwave Anisotropy Probe $($ WMAP) data $(\approx 13.7$ Gyr, Spergel et al. 2003; Dunkley et al. 2009), although they are still consistent with each other within the errors. However, the average MSTO age becomes $12.2 \pm 0.5 \mathrm{Gyr}$ when the CG97 values are used, reducing tension between stellar and WMAPbased ages. In addition, the age estimates based on the CG97 scale exhibit a steeper age-metallicity relation. Although only five clusters are included in the analysis, Figure 27 demonstrates that a different cluster $[\mathrm{Fe} / \mathrm{H}]$ scale could lead to a different

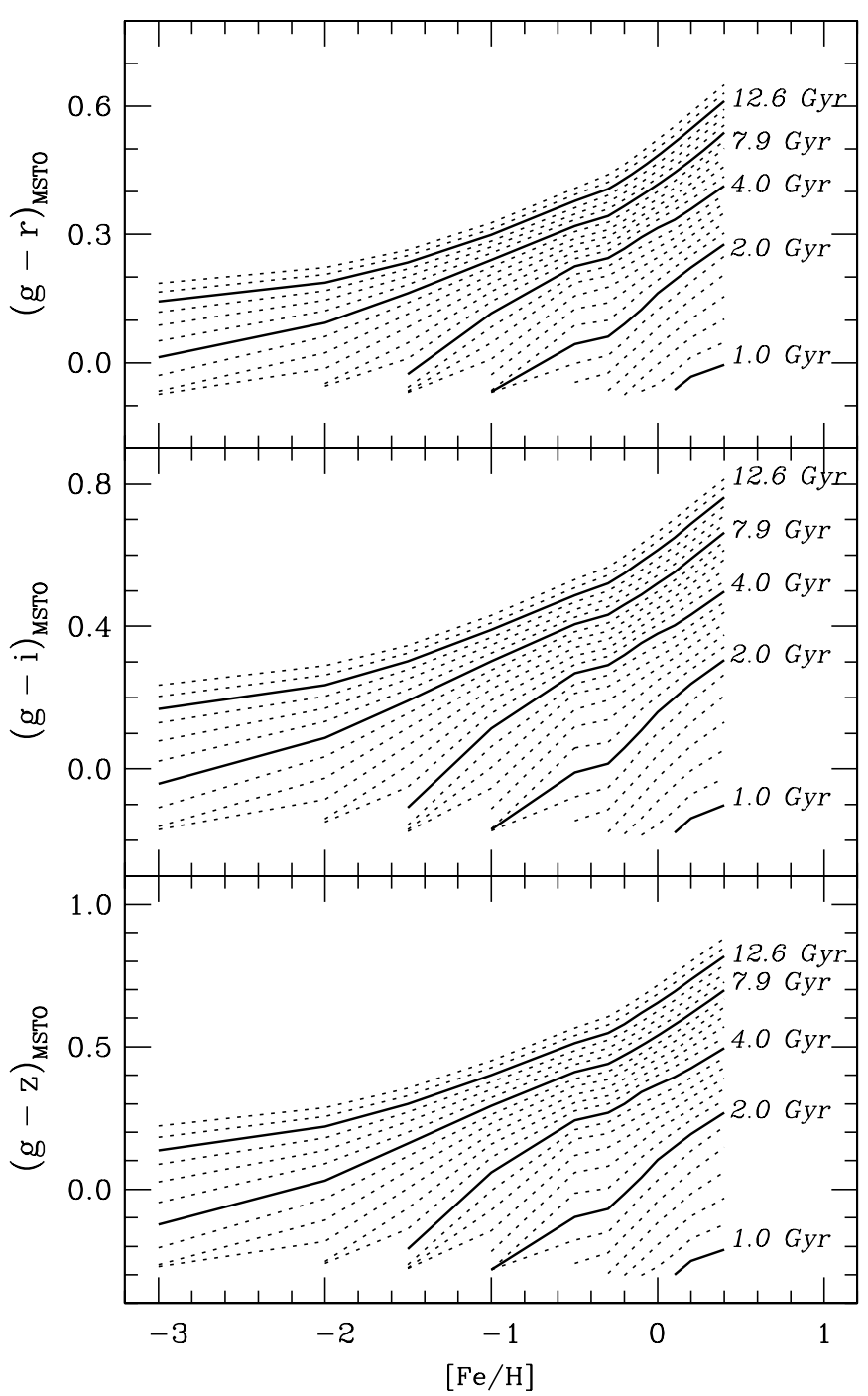

Figure 28. Theoretical MSTO colors in three color indices.

chemical enrichment scenario for the Galactic globular cluster system.

Finally, theoretical colors of the MSTO are presented in Figure 28 for three color indices, to aid the interpretation of CMDs in SDSS, as well as for future imaging surveys such as the Panoramic Survey Telescope \& Rapid Response System (PanSTARRS; Kaiser et al. 2002) and the Large Synoptic Survey Telescope (LSST; Ivezic et al. 2008a), which will use similar ugriz filter system. No empirical corrections were applied to the theoretical colors.

\section{SUMMARY AND DISCUSSION}

We generated a set of stellar isochrones in the SDSS ugriz system and performed an extensive test of these models for MS stars. We found that the models match observed cluster sequences within the errors of the adopted cluster parameters, for CMDs using $g-r, g-i$, and $g-z$ as color indices. These models correctly predict stellar colors and magnitudes over a wide range of metallicity, given the size of errors in distance, reddening, metallicity, and photometric calibration. However, we found $\sim 0.05-0.10$ mag offsets in $u-g$, indicating a problem in the model atmospheres for the $u$ bandpass. More accurate tests will be possible when future astrometric missions 
such as Gaia (Perryman et al. 2001) provide improved distance measurements for these clusters.

On the other hand, we found a strong departure of the models from the open cluster observations of the lower MS stars. The problem with cool stars has long been noted in other photometric systems, such as in Johnson-Cousins bandpasses, and is presumably due to our limited knowledge on the opacities and/or the equations of state used in the models. From the observation of M67, we proposed empirical corrections on the model color $-T_{\text {eff }}$ relations to alleviate a large distance error to the cluster from MS fitting. Our tests indicate that isochrones with these corrections provide an improved fit to MS of NGC 6791. Since colors and magnitudes of stars change rapidly with metallicity, accurate photometry of open clusters in ugriz will be useful to explore metallicity-dependent corrections on color$T_{\text {eff }}$ relations in the future.

We also tested models from Padova+ATLAS9 (Girardi et al. 2004) and DSEP+PHOENIX (Dotter et al. 2008) using cluster fiducial sequences as for comparison with the YREC+MARCS models - the models provide reasonable fits to the observations generally, but exhibit strong departures for the lower MS at solar and super-solar metallicities. However, Padova+ATLAS9 models do not include $\alpha$-element enhancement, prohibiting more accurate comparisons with cluster observation. In addition to $u-g$, we also found a large offset in $g-z$ for DSEP+PHOENIX models. In general, our YREC+MARCS models better match observed MSs than these models.

We derived distances and MSTO ages for our sample of Galactic globular clusters using our theoretical stellar isochrones. We performed isochrone fits for three color indices $(g-r, g-i$, and $g-z)$ over a wide wavelength range to constrain these quantities and to assess the size of internal systematic errors. Our distance estimates are consistent with those from Hipparcos-based subdwarf fitting, with an error of $\sigma_{(m-M)} \sim 0.03-0.11 \mathrm{mag}$ for individual clusters. We also found good agreement of our age estimates with those in the literature, with $\sigma_{\text {age }} \sim 10 \%-15 \%$. Our results demonstrate the ability to derive distances and ages for stars observed in SDSS, as well as in the future from imaging surveys such as Pan-STARRS (Kaiser et al. 2002) and with LSST (Ivezic et al. 2008a), which will employ similar ugriz filter systems.

We thank the referee for careful and detailed comments. D.A. thanks Grant Newsham for kindly proving his isochrone interpolation code. We thank Aaron Dotter and Brian Chaboyer for helpful discussions on the model comparison. D.A. gratefully acknowledges the support from the Presidential Fellowship in the Ohio State University. F.D. is supported by ANR (ANR-06-CIS6-009-01). T.C.B. is grateful for support in part by from grant AST 07-07776, and grants PHY 02-16783 and PHY 08-22648: Physics Frontiers Center/Joint Institute for Nuclear Astrophysics (JINA), awarded by the U.S. National Science Foundation. Any opinion, finding, and conclusions or recommendations expressed in this material are those of the author and do not necessarily reflect the views of the National Science Foundation. M.H.P. and D.M.T. acknowledge support from NSF grant AST-0807308. J.A.J. and T.M. acknowledge support from NSF grant AST-0707948. Ž.I. acknowledges support by NSF grant AST 07-07901.

Funding for the SDSS and SDSS-II has been provided by the Alfred P. Sloan Foundation, the Participating Institutions, the National Science Foundation, the U.S. Department of Energy, the National Aeronautics and Space Administration, the Japanese Monbukagakusho, the Max Planck Society, and the Higher Education Funding Council for England. The SDSS Web site is http://www.sdss.org/.

The SDSS is managed by the Astrophysical Research Consortium for the Participating Institutions. The Participating Institutions are the American Museum of Natural History, Astrophysical Institute Potsdam, University of Basel, University of Cambridge, Case Western Reserve University, University of Chicago, Drexel University, Fermilab, the Institute for Advanced Study, the Japan Participation Group, Johns Hopkins University, the Joint Institute for Nuclear Astrophysics, the Kavli Institute for Particle Astrophysics and Cosmology, the Korean Scientist Group, the Chinese Academy of Sciences (LAMOST), Los Alamos National Laboratory, the Max-PlanckInstitute for Astronomy (MPIA), the Max-Planck-Institute for Astrophysics (MPA), New Mexico State University, Ohio State University, University of Pittsburgh, University of Portsmouth, Princeton University, the United States Naval Observatory, and the University of Washington.

\section{REFERENCES}

Abazajian, K., et al. 2003, AJ, 126, 2081

Abazajian, K., et al. 2004, AJ, 128, 502

Abazajian, K., et al. 2005, AJ, 129, 1755

Adelman-McCarthy, J. K., et al. 2006, ApJS, 162, 38

Adelman-McCarthy, J. K., et al. 2007, ApJS, 172, 634

Adelman-McCarthy, J. K., et al. 2008, ApJS, 175, 297

Alexander, D. R., \& Ferguson, J. W. 1994, ApJ, 437, 879

Alonso, A., Arribas, S., \& Martínez-Roger, C. 1995, A\&A, 297, 197

Alonso, A., Arribas, S., \& Martínez-Roger, C. 1996, A\&AS, 117, 227

An, D., Terndrup, D. M., \& Pinsonneault, M. H. 2007a, ApJ, 671, 1640

An, D., Terndrup, D. M., Pinsonneault, M. H., Paulson, D. B., Hanson, R. B., \& Stauffer, J. R. 2007b, ApJ, 655, 233

An, D., et al. 2008, ApJS, 179, 326

Badnell, N. R., Bautista, M. A., Butler, K., Delahaye, F., Mendoza, C., Palmeri, P., Zeippen, C. J., \& Seaton, M. J. 2005, MNRAS, 360, 458

Bahcall, J. N., Pinsonneault, M. H., \& Basu, S. 2001, ApJ, 555, 990

Bessell, M. S. 1990, PASP, 102, 1181

Bessell, M. S., Castelli, F., \& Plez, B. 1998, A\&A, 333, 231

Bolte, M., \& Hogan, C. J. 1995, Nature, 376, 399

Cardelli, J. A., Clayton, G. C., \& Mathis, J. S. 1989, ApJ, 345, 245

Carraro, G., Villanova, S., Demarque, P., McSwain, M. V., Piotto, G., \& Bedin, L. R. 2006, ApJ, 643, 1151

Carretta, E., \& Gratton, R. G. 1997, A\&AS, 121, 95 (CG97)

Carretta, E., Gratton, R. G., Clementini, G., \& Fusi Pecci, F. 2000, ApJ, 533, 215 (C00)

Castelli, F., Gratton, R. G., \& Kurucz, R. L. 1997, A\&A, 318, 841

Castelli, F., \& Kurucz, R. L. 2003, in IAU Symp. 210, Modeling of Stellar Atmospheres, ed. N. E. Piskunov, W. W. Weiss, \& D. F. Gray (San Francisco, CA: ASP), poster A20 on enclosed CD-ROM (arXiv:astro-ph/0405087)

Chaboyer, B., Demarque, P., Kernan, P. J., Krauss, L. M., \& Sarajedini, A. 1996a, MNRAS, 283, 683

Chaboyer, B., Demarque, P., Kernan, P. J., \& Krauss, L. M. 1998, ApJ, 494, 96 Chaboyer, B., Demarque, P., \& Sarajedini, A. 1996b, ApJ, 459, 558

Chaboyer, B., Fenton, W. H., Nelan, J. E., Patnaude, D. J., \& Simon, F. E. 2001, ApJ, 562, 521

Chaboyer, B., \& Krauss, L. M. 2002, ApJ, 567, L45

Clem, J. L. 2006, PhD thesis, Univ. of Victoria

Clem, J. L., VandenBerg, D. A., \& Stetson, P. B. 2008, AJ, 135, 682

Davis, D. S., Richer, H. B., Anderson, J., Brewer, J., Hurley, J., Kalirai, J. S., Rich, R. M., \& Stetson, P. B. 2008, AJ, 135, 2155

De Angeli, F., Piotto, G., Cassisi, S., Busso, G., Recio-Blanco, A., Salaris, M., Aparicio, A., \& Rosenberg, A. 2005, AJ, 130, 116

de Bruijne, J. H. J., Hoogerwerf, R., \& de Zeeuw, P. T. 2001, A\&A, 367, 111

Delahaye, F., \& Pinsonneault, M. H. 2006, ApJ, 649, 529

Demarque, P., Guenther, D. B., Li, L. H., Mazumdar, A., \& Straka, C. W. 2007, Ap\&SS, 316, 31

Dotter, A., Chaboyer, B., Jevremović, D., Kostov, V., Baron, E., \& Ferguson, J. W. 2008, ApJS, 178, 89

Dunkley, J., et al. 2009, ApJS, 180, 306

Eggen, O. J., \& Sandage, A. R. 1959, MNRAS, 119, 255 
Eisenstein, D. J., et al. 2006, ApJS, 167, 40

ESA. 1997, The Hipparcos and Tycho Catalogues (ESA SP-1200) (Noordwijk: ESA)

Fornal, B., Tucker, D. L., Smith, J. A., Allam, S. S., Rider, C. J., \& Sung, H 2007, AJ, 133, 1409

Fukugita, M., Ichikawa, T., Gunn, J. E., Doi, M., Shimasaku, K., \& Schneider, D. P. 1996, AJ, 111, 1748

Geffert, M., \& Maintz, G. 2000, A\&AS, 144, 227

Girardi, L., Bertelli, G., Bressan, A., Chiosi, C., Groenewegen, M. A. T., Marigo, P., Salasnich, B., \& Weiss, A. 2002, A\&A, 391, 195

Girardi, L., Bressan, A., Bertelli, G., \& Chiosi, C. 2000, A\&AS, 141, 371

Girardi, L., Grebel, E. K., Odenkirchen, M., \& Chiosi, C. 2004, A\&A, 422, 205

Gratton, R., Bragaglia, A., Carretta, E., \& Tosi, M. 2006, ApJ, 642, 462

Gratton, R. G., Fusi Pecci, F., Carretta, E., Clementini, G., Corsi, C. E., \& Lattanzi, M. 1997, ApJ, 491, 749

Grevesse, N., \& Sauval, A. J. 1998, Space Sci. Rev., 85, 161

Grundahl, F., Stetson, P. B., \& Andersen, M. I. 2002, A\&A, 395, 481

Gunn, J. E., et al. 1998, AJ, 116, 3040

Gunn, J. E., et al. 2006, AJ, 131, 2332

Gustafsson, B., Edvardsson, B., Eriksson, K., Graae Jorgensen, U., Nordlund, A., \& Plez, B. 2008, A\&A, 486, 951

Hansen, B. M. S., et al. 2002, ApJ, 574, L155

Harris, W. E. 1996, AJ, 112, 1487

Hauschildt, P. H., Allard, F., \& Baron, E. 1999, ApJ, 512, 377

Heasley, J. N., \& Christian, C. A. 1986, ApJ, 307, 738

Hogg, D. W., Finkbeiner, D. P., Schlegel, D. J., \& Gunn, J. E. 2001, AJ, 122, 2129

Holberg, J. B., \& Bergeron, P. 2006, AJ, 132, 1221

Iglesias, C. A., \& Rogers, F. J. 1996, ApJ, 464, 943

Ivezic, Z., Tyson, J. A., Allsman, R., Andrew, J., Angel, R., \& for the LSST Collaboration 2008a, arXiv:0805.2366

Ivezić, Ž., et al. 2004, Astron. Nachr., 325, 583

Ivezić, Ž., et al. 2008b, ApJ, 684, 287

Jensen, E., Boesgaard, A. M., \& Deliyannis, C. P. 2006, BAAS, 38, 1134

Jimenez, R., \& Padoan, P. 1998, ApJ, 498, 704

Johnson, H. L. 1957, ApJ, 126, 121

Jurić, M., et al. 2008, ApJ, 673, 864

Kaiser, N., et al. 2002, Proc. SPIE, 4836, 154

Kim, Y.-C., Demarque, P., Yi, S. K., \& Alexander, D. R. 2002, ApJS, 143, 499

Kirby, E. N., Guhathakurta, P., \& Sneden, C. 2008, ApJ, 682, 1217

Korn, A. J., Grundahl, F., Richard, O., Barklem, P. S., Mashonkina, L., Collet, R., Piskunov, N., \& Gustafsson, B. 2006, Nature, 442, 657

Korn, A. J., Grundahl, F., Richard, O., Mashonkina, L., Barklem, P. S., Collet, R., Gustafsson, B., \& Piskunov, N. 2007, ApJ, 671, 402

Kraft, R. P., \& Ivans, I. I. 2003, PASP, 115, 143 (KI03)

Kraft, R. P., \& Ivans, I. I. 2004, in Carnegie Observatories Astrophysics Ser. 4, Origin and Evolution of the Elements, ed. A. McWilliam \& M. Rauch (Pasadena, CA: Carnegie Observatories), http://www.ociw.edu/ociw/ symposia/series/symposium4/proceedings.html

Krauss, L. M., \& Chaboyer, B. 2003, Science, 299, 65

Lejeune, Th., Cuisinier, F., \& Buser, R. 1997, A\&AS, 125, 229

Lejeune, Th., Cuisinier, F., \& Buser, R. 1998, A\&AS, 130, 65

Lupton, R. H., Gunn, J. E., \& Szalay, A. S. 1999, AJ, 118, 1406
Lupton, R. H., Ivezic, Z., Gunn, J. E., Knapp, G., Strauss, M. A., \& Yasuda, N. 2002, Proc. SPIE, 4836, 350

Michaud, G., Richard, O., Richer, J., \& VandenBerg, D. A. 2004, ApJ, 606, 452

Mihalas, D., Hummer, D. G., Mihalas, B. W., \& Daeppen, W. 1990, ApJ, 350, 300

Oke, J. B., \& Gunn, J. E. 1983, ApJ, 266, 713

Origlia, L., Valenti, E., Rich, R. M., \& Ferraro, F. R. 2006, ApJ, 646, 499

Perryman, M. A. C., et al. 2001, A\&A, 369, 339

Pier, J. R., Munn, J. A., Hindsley, R. B., Hennessy, G. S., Kent, S. M., Lupton, R. H., \& Ivezić, Ž. 2003, AJ, 125, 1559

Pinsonneault, M. H., Terndrup, D. M., Hanson, R. B., \& Stauffer, J. R. 2003, ApJ, 598,588

Pinsonneault, M. H., Terndrup, D. M., Hanson, R. B., \& Stauffer, J. R. 2004, ApJ, 600, 946

Reid, I. N. 1997, AJ, 114, 161

Reid, N. 1998, AJ, 115, 204

Renzini, A. 1991, in NATO ASIC Proc. 348: Observational Tests of Cosmological Inflation ed. T. Shanks, A. J. Banday, \& R. S. Ellis (Dordrecht: Kluwer), 13

Renzini, A., et al. 1996, ApJ, 465, L23

Rider, C. J., Tucker, D. L., Smith, J. A., Stoughton, C., Allam, S. S., \& Neilsen, E. H., Jr. 2004, AJ, 127, 2210

Rogers, F. J., \& Nayfonov, A. 2002, ApJ, 576, 1064

Rosenberg, A., Saviane, I., Piotto, G., \& Aparicio, A. 1999, AJ, 118, 2306

Salaris, M., Chieffi, A., \& Straniero, O. 1993, ApJ, 414, 580

Salaris, M., \& Weiss, A. 2002, A\&A, 388, 492

Sandage, A. 1970, ApJ, 162, 841

Sandquist, E. L. 2004, MNRAS, 347, 101

Saumon, D., Chabrier, G., \& van Horn, H. M. 1995, ApJS, 99, 713

Schlegel, D. J., Finkbeiner, D. P., \& Davis, M. 1998, ApJ, 500, 525

Searle, L., \& Zinn, R. 1978, ApJ, 225, 357

Sills, A., Pinsonneault, M. H., \& Terndrup, D. M. 2000, ApJ, 534, 335

Smith, J. A., et al. 2002, AJ, 123, 2121

Sollima, A., Beccari, G., Ferraro, F. R., Fusi Pecci, F., \& Sarajedini, A 2007, MNRAS, 380, 781

Spergel, D. N., et al. 2003, ApJS, 148, 175

Stetson, P. B. 1987, PASP, 99, 191

Stetson, P.-B. 1994, PASP, 106, 250

Stetson, P. B., \& Harris, W. E. 1988, AJ, 96, 909

Stetson, P. B., Vandenberg, D. A., \& Bolte, M. 1996, PASP, 108, 560

Stoughton, C., et al. 2002, AJ, 123, 485

Straniero, O., Chieffi, A., \& Limongi, M. 1997, ApJ, 490, 425

Tucker, D. L., et al. 2006, Astron. Nachr., 327, 821

VandenBerg, D. A. 2000, ApJS, 129, 315

VandenBerg, D. A., \& Clem, J. L. 2003, AJ, 126, 778

VandenBerg, D. A., Richard, O., Michaud, G., \& Richer, J. 2002, ApJ, 571, 487

Vandenberg, D. A., Stetson, P. B., \& Bolte, M. 1996, ARA\&A, 34, 461

Venn, K. A., Irwin, M., Shetrone, M. D., Tout, C. A., Hill, V., \& Tolstoy, E. 2004, AJ, 128, 1177

York, D. G., et al. 2000, AJ, 120, 1579

Zinn, R., \& West, M. J. 1984, ApJS, 55, 45

Zoccali, M., et al. 2001, ApJ, 553, 733 